Draft VERsion July 22, 2015

Preprint typeset using $\mathrm{LT}_{\mathrm{E}} \mathrm{X}$ style emulateapj v. 5/2/11

\title{
OBSERVATIONS OF EXOPLANET ATMOSPHERES
}

\author{
IAN J. M. CROSSFIELD ${ }^{1,2}$ \\ Draft version July 22, 2015
}

\begin{abstract}
Detailed characterization of an extrasolar planet's atmosphere provides the best hope for distinguishing the makeup of its outer layers, and the only hope for understanding the interplay between initial composition, chemistry, dynamics \& circulation, and disequilibrium processes. In recent years, some areas have seen rapid progress while developments in others have come more slowly and/or have been hotly contested. This article gives an observer's perspective on the current understanding of extrasolar planet atmospheres prior to the considerable advances expected from the next generation of observing facilities. Atmospheric processes of both transiting and directly-imaged planets are discussed, including molecular and atomic abundances, cloud properties, thermal structure, and planetary energy budgets. In the future we can expect a continuing and accelerating stream of new discoveries, which will fuel the ongoing exoplanet revolution for many years to come.
\end{abstract}

\section{INTRODUCTION}

The exoplanet revolution is well underway. The last decade has seen order-of-magnitude increases in the number of known planets beyond the Solar system. Surveys reveal that while hot Jupiters on few-day orbits occur around $\lesssim 1 \%$ of Sunlike stars (Marcy et al. 2005, Howard et al. 2012); smaller, cooler sub-Neptunes and superEarths occur far more frequently (Dong \& Zhu 2013; Fressin et al. 2013), and are $2-3 \times$ more common still around low-mass stars (Bonfils et al. 2013, Mulders et al. 2015: Dressing \& Charbonneau 2015). At larger orbital separations planets with super-Jovian masses are rare, occurring around $<5 \%$ of stars (Biller et al. 2013).

Even greater than the advances made in planetary demographics is the dramatic progress made toward understanding the atmospheres of these distant worlds. Detailed characterization of a planet's atmosphere provides the best hope for distinguishing the makeup of its outer layers, and the only hope for understanding the interplay between initial composition (Madhusudhan et al. 2011b Mordasini et al. 2012), chemistry (Fortney et al. 2010, Barman et al. 2011a), dynamics \& circulation (Showman et al. 2009), and disequilibrium processes (Moses et al. 2013 Line \& Yung 2013). Mass and radius measurements alone cannot uniquely identify the $\mathrm{H}_{2}$, ice, and rock content of many planets (Adams et al. 2008). Atmospheric measurements are essential.

Given the rapid expansion of the field, a steady stream of updated reviews is required to keep up to date. A number of excellent review articles have been published on the topic of exoplanet atmospheres, and much in these remains accurate and relevant (Marley et al. 2007; Burrows et al. 2010, Seager \& Deming |2010; Madhusudhan et al. 2014; Burrows 2014b; Bailey 2014). Since the latest of these do not discuss the most recent exciting developments, while others discuss results now seen to have been illusory, the time is ripe for another comprehensive review.

\footnotetext{
${ }^{1}$ Lunar \& Planetary Laboratory, University of Arizona Lunar, 1629 E. University Blvd., Tucson, AZ, USA ianc@lpl.arizona.edu

${ }^{2}$ NASA Sagan Fellow
}

This article gives one observer's perspective of our current understanding of extrasolar planet atmospheres. The document is organized as follows: observing methods and the modeling approaches used to infer atmospheric properties are described in Secs. 2 and 3 , respectively. Sec. 4 summarizes the status of measurements of atmospheric composition and chemistry, leading in to a discussion of clouds and hazes in Sec. 5 and circulation, energy budgets, and variability in Sec. 6. Finally, Sec. 7 concludes with a look toward the exciting future of the field.

\section{EXOPLANET OBSERVING TECHNIQUES}

Before diving in to the many recent advances in the field, it is worthwhile to review the various observational techniques used to obtain the necessary data. This section begins with direct imaging in Sec. 2.1. then moves to shorter-period (typically transiting) planets: occultation studies in Sec. 2.2 phase curves in Sec. 2.3, and highdispersion spectroscopy in Sec. 2.4. Fig. 1 schematically depicts each of these approaches.

\subsection{Direct imaging}

Direct imaging - spatially resolving a planet at some angular separation from its host star - is a challenging endeavor. At a distance of $10 \mathrm{pc}$ a planet in a Jupiter-like orbit ( $a=5 \mathrm{AU})$ is just 0.5" from its host star, well within the $\sim 1$ " angular resolution available to most ground-based telescopes. Only with adaptive optics (Hardy 1998) can these facilities reach their theoretical, diffraction-limited angular resolution of $0.2 "(\lambda / \mu \mathrm{m})(D / m)^{-1}$. AO-equipped or space-based telescopes can therefore resolve extrasolar planets on wide orbits, but even for young, hot planets still glowing from the heat of their formation the planets are at best $\lesssim 10^{-4}$ times as bright as the nearby host star. Adaptive optics or space-based facilities are essential to produce diffraction-limited images, but even fairly faint levels of residual scattered starlight must be removed before any faint planet can be seen. Fig 2 shows the current state of the art relative to a variety of known planets.

The art and science of direct imaging focuses to a considerable degree on removing the residual starlight 


\section{Eclipse:}

Removing "star" from "star plus planet" flux reveals the planet's thermal emission or albedo:

\section{Direct Imaging:}

Spatially resolving planet from star allows measurement of thermal emission or albedo.

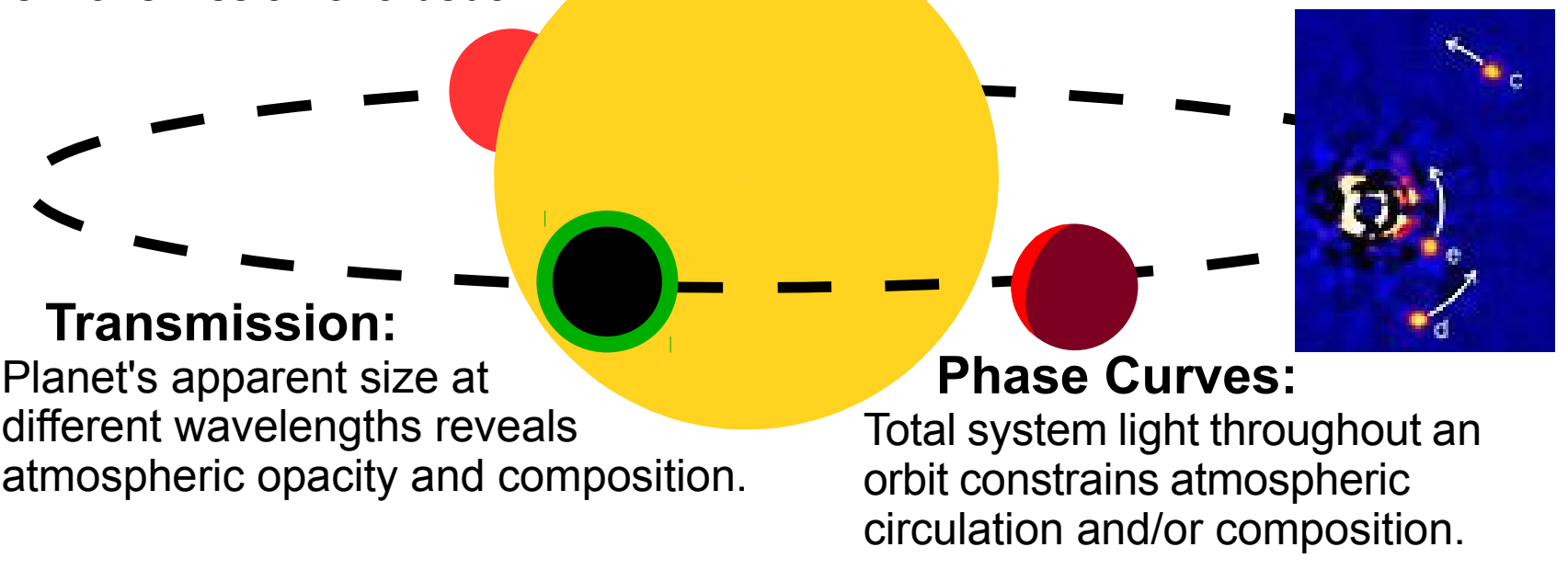

FIG. 1.- Observational approaches used to characterize the atmospheres of extrasolar planets.

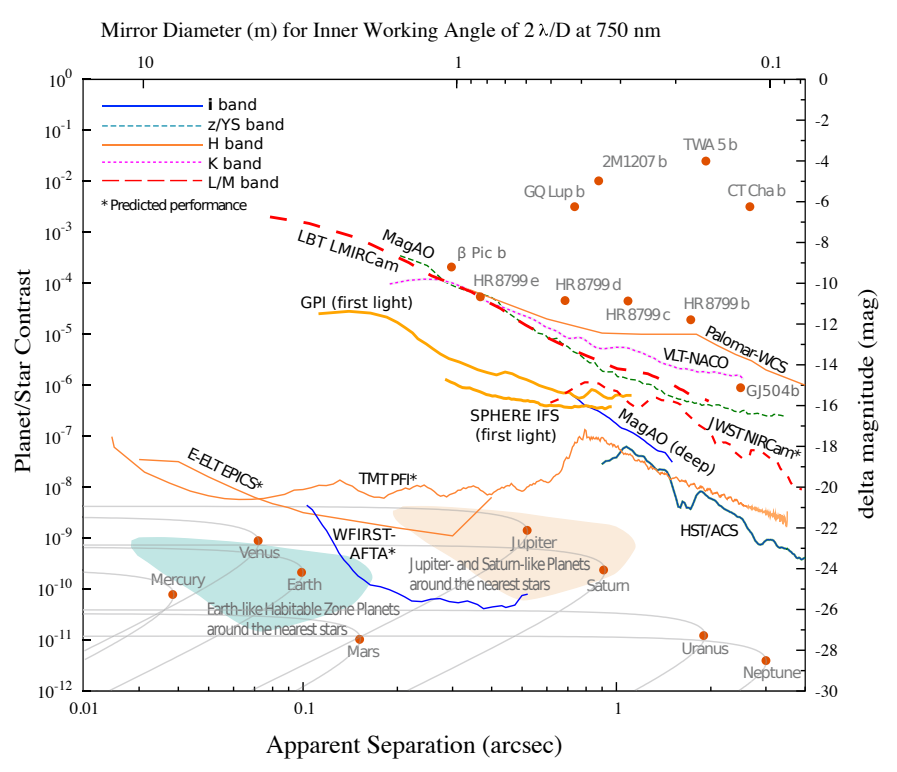

FIG. 2.- Planet/star contrast achieved via current and planned direct imaging instruments. Orange points indicate a subset of detected planets (at upper right, as seen in $K$ band), and Solar System planets at 10 pc (bottom, assuming reflected light). Updated from Mawet et al. (2012), with additional data added (Close et al. 2014 Macintosh et al.|2014, Kuzuhara et al. 2013 Skemer et al. 2014b Spergel et al. 2015, Males et al., in prep., and private communication from A. Vigan).

as effectively as possible. Steady progress has been made since the first detections (Chauvin et al. 2004, Marois et al. 2008). A variety of techniques including simultaneous differential imaging (Smith 1987; Rosenthal et al. 1996), spectral differential imaging ( \& Ford 2002), angular differential imaging (Marois et al. 2006), and others have been developed; see Mawet et al. (2012) for a review. Whatever the approach, observations taken with either successive photometric filters or a spectrograph then measure the planet's thermal emission. In principle reflected light could also be detected from these planets, but the planet-star contrast ratios are much lower: $\left(2 \times 10^{-7}\right) A_{G}\left(R_{p} / R_{J}\right)^{2}(a / \mathrm{AU})^{-2}$, where $A_{G}$ is the planet's geometric albedo. Nonetheless future ground- and space-based telescopes will study some small subset of giant planets in reflected light (Crossfield 2013, Quanz et al. 2015, Marley et al. 2014, Burrows 2014a; $\mathrm{Hu}$ 2014).

Similar to directly-imaged planets but easier to study are solivagant (free-floating) or widely-separated planetary-mass objects. Whether these objects form like traditional planets is as yet undetermined (see the discussion in Beichman et al. 2014a), but it is clear that these wanderers can also provide key insights into atmospheric properties of cool planetary atmospheres. These have no nearby host star and so can be characterized even by seeing-limited observations (without the need for adaptive optics), just as brown dwarfs have been studied for several decades. A further advantage of any type of direct imaging is that once the necessary contrast has been reached, one obtains much higher $\mathrm{S} / \mathrm{N}$ and spectral resolution than is possible for a transiting planet of comparable brightness. This is because photon shot noise is much reduced by suppressing the host starlight and planets are visible all night, every night rather than for just a few hours per orbit.

\subsection{Transits and Eclipses}

Transiting planets occult their host stars, resulting in a periodic flux decrement approximately equal to $\left(R_{P} / R_{*}\right)^{2}$ - this is $10^{-4}$ for Earth orbiting the Sun, but is $2 \times 10^{-3}$ for a $1.5 R_{\oplus}$ super-Earth orbiting an M4 dwarf and $1-2 \%$ for a typical hot Jupiter. When the planet has an atmosphere, its atmospheric opacity varies with wavelength and so the radius at which the planet's atmosphere becomes optically thick changes with wavelength (Seager 
\& Sasselov 2000, Brown et al. 2001, Hubbard et al. 2001). One therefore measures the flux decrement at multiple wavelengths; the observed quantity $\left(R_{P}(\lambda) / R_{*}\right)^{2}$ is termed the "transmission spectrum." At moderate spectral resolution, its spectral features have amplitudes of a few times $H R_{P} / R_{*}^{2}$ (Miller-Ricci et al. 2009), where $H$ is the atmospheric scale height. Thus to measure transmission features in an Earth-Sun twin system requires a fractional precision approaching $10^{-6}(1 \mathrm{ppm})$, but this increases to $\sim 15 \mathrm{ppm}$ for our $\mathrm{H}_{2}$-rich super-Earth orbiting its $\mathrm{M}$ star and even larger for hot Jupiters. (In fact, both $\mathrm{S} / \mathrm{N}$ considerations and refractive effects limit the useful study of habitable atmospheres in transit to $\mathrm{M}$ star systems; Bétrémieux \& Kaltenegger 2014). Clouds and hazes tend to mute spectral features from molecular and atomic absorbers, producing nearly flat transmission spectra (Seager \& Sasselov 2000; Marley et al.|2013).

Transiting planets on circular orbits also pass behind their host stars, blocking the planets' thermal emission and decreasing the flux observed from the systems by $\left(R_{P} / R_{*}\right)^{2} F_{\nu, P} / F_{\nu, *}$, where $F_{\nu}$ is the emergent flux density of the planet or star. At longer wavelengths $F_{\nu, P}$ is dominated by thermal emission resulting from the planet's internal heat and/or reprocessed starlight; at shorter wavelengths scattered starlight may also be a significant contribution. Assuming simple, blackbodylike emission spectra, at $15 \mu \mathrm{m}$ the eclipse signal is just $0.8 \mathrm{ppm}$ for an Earth-Sun twin system, 30 ppm for the super-Earth orbiting an M dwarf described above, and a few times $10^{-3}$ for hot Jupiters. To characterize atmospheric features rather than merely detect planetary emission requires perhaps a further tenfold improvement in precision. Planetary emission or reflection can also be studied via phase curves (Sec. 2.3. . Fig. 1 demonstrates how transits, eclipses, and phase curves all provide complementary insights into a transiting planet's atmosphere.

Most observations to date have employed lowdispersion spectroscopy or broadband photometry. An alternative but less-explored method of transmission observations, using high-dispersion spectroscopy from large-aperture telescopes, is discussed below in Sec. 2.4. The most profitable tool for studying transiting exoplanet atmospheres has been space-based spectroscopy with HST, which has been used to study the atmospheres of a rapidly-growing number of both hot Jupiters and sub-Jovians (Charbonneau et al. 2002; Lecavelier Des Etangs et al. 2008a; Evans et al. 2013; Kreidberg et al. 2014b Knutson et al. 2014a). Observations with Spitzer and ground-based telescopes have also been frequently employed (Knutson et al. 2008; Désert et al. 2011, Croll et al. 2011) but with typically less secure characterization of atmospheric properties. JWST will soon be the superlative instrument for these studies, providing over $10 \times$ the total spectral range of HST/WFC3, with a sensitivity $\sim 3 \times$ greater than HST and $8 \times$ greater than Spitzer (Beichman et al. 2014c).

\subsection{Phase curves}

Phase curves track the modulation of planetary thermal emission and/or reflected starlight throughout a planet's orbit. These observations complement secondary eclipses, which only measure emission from a transiting planet's day side. Phase curves provide a wealth of information about planetary atmospheric dynamics and energetics by measuring longitudinal brightness temperature maps, thereby constraining atmospheric conditions across the planet's surface. The phase curve amplitude determines the day-night temperature contrast in the thermal infrared, or constrains the planet's albedo at shorter wavelengths. The phase offset measures the longitude of the planet's brightest or dimmest point relative to the substellar meridian.

Phase curve morphologies are in general a degenerate outcome an atmosphere's radiative, advective, and drag timescales and the efficiency with which winds circulate incident stellar energy around the planet. If the incident stellar flux is immediately re-radiated, the hottest point on the planet is at the substellar point (which receives the highest level of incident flux). Nonzero infrared phase offsets thus imply heat transport around the planet. Clouds or hazes can move the infrared photosphere to higher altitude, resulting in a larger observed temperature contrast and smaller phase offset (Pont et al. 2013). Large phase offsets could indicate strong advection or drag forces (Cowan \& Agol 2011, Perez-Becker \& Showman 2013) or re-radiation of thermal energy deposited by shock tronts in the planet's atmosphere where supersonic equatorial jets transition to subsonic speeds (Heng 2012). Shocks and supersonic flows may be absent if magnetic drag forces are significant. Non-negligible Lorentz forces may occur in the presence of planetary magnetic fields when alkali atoms are ionized (Perna et al. 2010, Rauscher \& Menou 2013).

To obtain a phase curve, one observes a system for some substantial fraction of an orbit (Knutson et al. 2007). If the planet is transiting, one or more secondary eclipses provides an absolute reference to disentangle stellar flux from combined "star plus planet" light. If the planet does not transit, the resulting constraints on atmospheric properties are looser (Cowan et al. 2007, Crossfield et al. 2010). While a phase curve provides substantially more information than a secondary eclipse, the former requires considerably more observing time and is more susceptible to degradation by slowly-varying, lowamplitude instrumental systematics (Agol et al. 2010, Crossfield et al. 2012c).

\subsection{High-dispersion spectroscopy}

Ground-based telescopes permit large, massive spectrographs with $\lambda / \Delta \lambda \gtrsim 30,000$, vs. a few thousand at most for HST or JWST. The increased spectral resolution dramatically increases the contrast of absorption features in exoplanetary spectra, more than making up for the increased calibration challenges resulting from ground-based spectroscopy.

When applied to short-period hot Jupiters, highdispersion spectroscopy treats planet-star systems as double-lined spectroscopic binaries. The technique now regularly detects thermal emission from hot Jupiters at the $\sim 10^{-4}$ level relative to the star, ascertains whether targets have a temperature inversion, and measures molecular abundances as small as $\sim 10^{-5}-10^{-4}$. This powerful new technique has produced a surge in the number of planets with robust molecular detections or constraints on atmospheric properties (Snellen et al. 2010; Crossfield et al. 2011; Brogi et al. 2012, 2013, 2014; Rodler et al. 2012, 2013, Birkby et al. 2013, de Kok et al. 

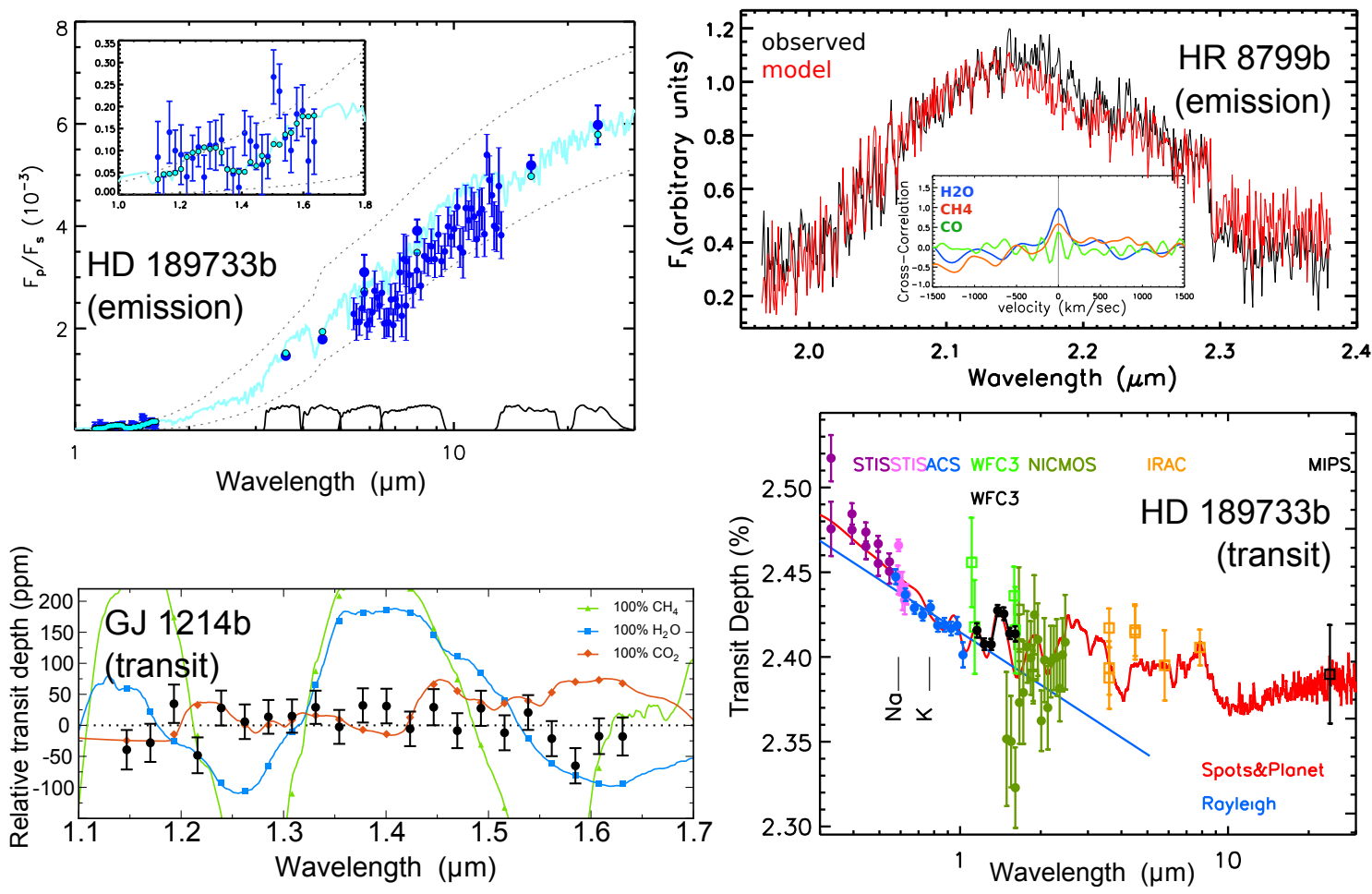

FIG. 3.- Some of the best-characterized exoplanets, highlighting the main atmospheric conclusions to date. Clockwise from upper left: approximately isothermal emission spectra of hot Jupiters with weak $\mathrm{H}_{2} \mathrm{O}$ absorption features (HD 189733b, emission); high-quality emission spectra of directly imaged planets, revealing $\mathrm{H}_{2} \mathrm{O}, \mathrm{CH}_{4}$, and $\mathrm{CO}$ ( $\mathrm{HR} 8799 \mathrm{~b}$ ); transmission spectra of hot Jupiters showing short-wavelength slopes, $\mathrm{H}_{2} \mathrm{O}$ at $1.4 \mu \mathrm{m}$, and no conclusive features at longer wavelengths (HD 189733b, transit); and flat, featureless transmission spectra of cool sub-Jovians, indicative of high-altitude haze (GJ 1214b). Figures adapted from Crouzet et al. (2014); Todorov et al. (2014); Barman et al. (2015); McCullough et al. (2014); Kreidberg et al. (2014b).

2013 Lockwood et al. 2014). A further strength of highdispersion Doppler spectroscopy is its applicability to non-transiting planetary systems, where high-dispersion observations break the $\sin i$ degeneracy that plagues interpretation of non-transiting phase variations.

When coupled to an AO system, high-dispersion spectroscopy can also be use to great effect to study directly imaged planets. This has been done for several planets at medium resolution (Konopacky et al. 2013, Barman et al. 2015) and at $\lambda / \Delta \lambda \sim 90,000$; for beta Pic b (Snellen et al. 2014). The latter type of observation directly constrains planetary rotation rates and could eventually place high-fidelity constraints on planets' thermal structure (Line et al. 2014a, 2015) and global 2D surface brightness profiles (Crossfield 2014).

\section{INFERRING ATMOSPHERIC PROPERTIES FROM OBSERVATIONS}

The previous section describes the observations used to constrain an exoplanet's atmosphere, but these data do not automatically provide atmospheric parameters. Only by comparison to physically relevant models can the parameters of interest - temperature, abundances, etc. - be inferred. Like the observations themselves, obtaining useful model-derived properties is challenging. In the simplest cases, toy models provide useful insights - constraining temperatures via comparison with blackbodies (Charbonneau et al. 2005; Deming et al. 2005), or deriving temperature and mean molecular weight from Rayleigh scattering slopes (Lecavelier Des Etangs et al. 2008b - but typically more elaborate models are needed.
Analyses of exoplanet atmospheres fall into two general categories: forward models, which assume a particular combination of parameters to generate an observable (e.g., a spectrum); and the inverse or "retrieval" approach, which explicitly aims to determine the bestfit parameters and their uncertainties from the available data. The recent review by Madhusudhan et al. (2014) discusses both of these techniques. Though sometimes seen as at odds with each other, the two approaches are complementary.

Forward models are essential to predict the observable properties of previously unobserved objects for which scant data exists. Such models have played a key role in focusing attention on especially interesting or easily observable atmospheric features: e.g., alkali absorption in hot Jupiters (Seager \& Sasselov 2000; Barman et al. 2002, Charbonneau et al. 2002). Forward models also often include more detailed treatments of atmospheric properties which would be too time-consuming to include in a retrieval; for example, dust formation and dispersal in substellar atmospheres (Helling et al. 2008).

Retrieval tools (Irwin et al. 2008 Madhusudhan \& Seager 2009, Benneke \& Seager 2012; Line et al. [2013, Cubillos et al., in prep.) necessarily include some form of forward model, incorporated within an algorithm that explores the atmospheric parameter phase space consistent with the measurements in hand. To adequately sample the atmospheric parameter posterior distributions in a timely manner various simplifications are often made: molecular abundances may be constant with altitude, thermal profiles may assume an arbitrary parametriza- 
tion without enforcement of radiative equilibrium, and so forth. However, techniques and computing power are quickly advancing to the point where many of these assumptions can already be relaxed (Line et al. 2014a 2015, Benneke 2015). In any case, the power of retrieval is that - subject to the model assumptions and the reliability of the data - the technique provides statistically robust confidence intervals on the desired parameters.

For whatever reason, retrieval analyses have focused mainly on transiting planets (with some exceptions; Lee et al. 2013) while analyses of directly imaged planets have relied mainly on forward models. Some of the latter studies compare observations to multidimensional grids of precomputed template spectra, though the variables are typically fewer than in the retrieval analyses described above. It seems likely that the field could benefit from the complementary perspectives that would be provided by application of retrieval methods to spectra of directly imaged planets.

\section{MOLECULES \& ATOMS}

One of the first questions asked about an exoplanet's atmosphere is, "What is it made of?" The last few years have seen rapid strides toward answering this question for a wide range of molecules and atomic species, as were seen in the preceding years for studies of brown dwarfs (e.g., Lodders \& Fegley 2006). This section first considers molecular detections from both spectroscopy and broadband photometry, and then discusses recent measurements of atoms and ions in planets' upper atmospheres. A comprehensive overview of recent detections is also given by Bailey (2014), and Heng et al. (2015) present an excellent discussion of the key concepts involved in atmospheric chemistry. Clouds and hazes are subsequently discussed in Sec. 5, and thermal structure in Sec. 6.4.

\subsection{Molecular Detections and Abundances}

Planetary atmospheres are composed primarily of molecules. The relative abundances of different molecular species depend on the complex interplay between bulk chemical composition resulting from the planet's formation \& evolution, differentiation, atmospheric circulation, and photochemical and other disequilibrium processes. Fig. 4 shows the expected abundances of various molecules in equilibrium (but see Sec. 4.3). For typical, solar-like abundances the 1000-2500 K temperatures of most giant exoplanets studied to date indicates that $\mathrm{CO}$ and $\mathrm{H}_{2} \mathrm{O}$ will be the most abundant and spectroscopically active species in equilibrium conditions (Burrows \& Sharp 1999, Lodders \& Fegley 2006). CO $_{2}$ will also be present in high metallicity environments at these warmer temperatures; its abundance remains lower than that of $\mathrm{CO}$ for all but the highest metallicities (e.g., Moses et al. 2013). Below $\sim 1300 \mathrm{~K} \mathrm{CH}_{4}$ should become more abundant than $\mathrm{CO}$, and below $\sim 700 \mathrm{~K} \mathrm{NH}_{3}$ become increasingly abundant as well (Burrows \& Sharp 1999). The following sections review the measurements of various molecules detected by either spectroscopy (Sec. 4.1.1) or broadband photometry (Sec. 4.1.2.

\subsubsection{Spectroscopically Resolved Molecules}

The most robust detections and measurements of extrasolar atmospheric molecular abundances come from observations that resolve either the individual lines, or (at lower dispersion) the overall shape of molecular bandheads.

$\mathbf{H}_{2}$ is of course the dominant constituent of most exoplanets studied to date. Transiting planets with masses from Doppler spectroscopy reveal planet densities that can only be fit with significant $\mathrm{H}_{2}$ envelopes (Fortney et al. 2007). Substantial $\mathrm{H}_{2}$ can also be inferred from the Rayleigh scattering slope of a planet's transmission spectrum (Lecavelier Des Etangs et al. 2008a b). This feature has been seen in both hot Jupiters and the $\sim 800 \mathrm{~K}$ Neptune-sized GJ 3470b (Pont et al. 2013; Nascimbeni et al. 2013, Biddle et al. 2014), though a recent analysis claims that starspots can masquerade as this Rayleigh scattering signal (McCullough et al. 2014; Oshagh et al. 2014). $\mathrm{H}_{2}$ also makes itself known via continuum-induced absorption (CIA), especially in the $H$ and $K$ bands (Borysow et al. 1997). The CIA feature is explicitly included when computing model spectra of all giant extrasolar planets, so $\mathrm{H}_{2}$ 's presence is implicit in these objects (as it is in planet evolutionary models). However, no study has yet used CIA to explicitly measure the atmospheric $\mathrm{H}_{2}$ mixing ratio.

CO is abundant in giant, hot exoplanet atmospheres, and its regularly-spaced rovibrational lines make it an especially easy molecule to detect at high dispersion. In this way, the molecule has been unambiguously detected in numerous hot Jupiters, though in most cases the detection alone is not sufficient to tightly constrain CO's atmospheric abundance (Snellen et al. 2010, Brogi et al. 2012, 2014, Rodler et al. 2012, 2013, Birkby et al. 2013). CO has also been observed in several directlyimaged planets at medium or low dispersion, including HR 8799b and c (Konopacky et al. 2013, Barman et al. 2015) and beta Pic b (Snellen et al. 2014). These studies generally reveal CO abundances consistent with Solar metallicity and equilibrium chemistry, though HR $8799 \mathrm{~b}$ intriguingly shows only $\sim 50 \%$ as much $\mathrm{CO}$ as predicted (Barman et al. 2015). In all these planets, the $\mathrm{CO} / \mathrm{CH}_{4}$ ratio is much higher than expected from equilibrium processes. This high $\mathrm{CO}$ abundance is interpreted as evidence for strong quenching of these species by vigorous vertical mixing (see Sec. 4.3.).

$\mathbf{H}_{2} \mathbf{O}$ is roughly as abundant as $\mathrm{CO}$ in the atmosphere (see Fig. 4), and indeed it sculpts their NIR spectra much more strongly than does CO. Again, medium- and high-dispersion spectroscopy has detected the species in a rapidly-growing number of directly imaging planets (e.g., Patience et al. 2010, Gagné et al. 2014, Bonnefoy et al. 2014a: Barman et al. 2015; Todorov et al. 2015) and in several hot Jupiters (Lockwood et al. 2014, Brogi et al. 2014 Birkby et al. 2013). Perhaps the largest body of $\mathrm{H}_{2} \mathrm{O}$ measurements comes from low-resolution spectroscopy of transiting systems using HST's WFC3/G141 grism spectrograph, which covers the $1.4 \mu \mathrm{m} \mathrm{H}_{2} \mathrm{O}$ bandhead. These observations reveal $\mathrm{H}_{2} \mathrm{O}$ absorption in many planets (e.g., Deming et al. 2013; Nikolov et al. 2015, Kreidberg et al. 2014a), albeit sometimes at low S/N. Similar spectroscopy with Spitzer's IRS spectrograph also hint at $\mathrm{H}_{2} \mathrm{O}$ absorption at $6 \mu \mathrm{m}$ (Grillmair et al. 2008; Todorov et al. 2014). The lowest-mass planet with observed $\mathrm{H}_{2} \mathrm{O}$ is the $\sim 1000 \mathrm{~K}$ Neptune-sized HAT-P-11b (Fraine et al. 2014). $\mathrm{H}_{2} \mathrm{O}$ mixing ratios for all characterized planets are plotted in Fig. 5. all reported abun- 

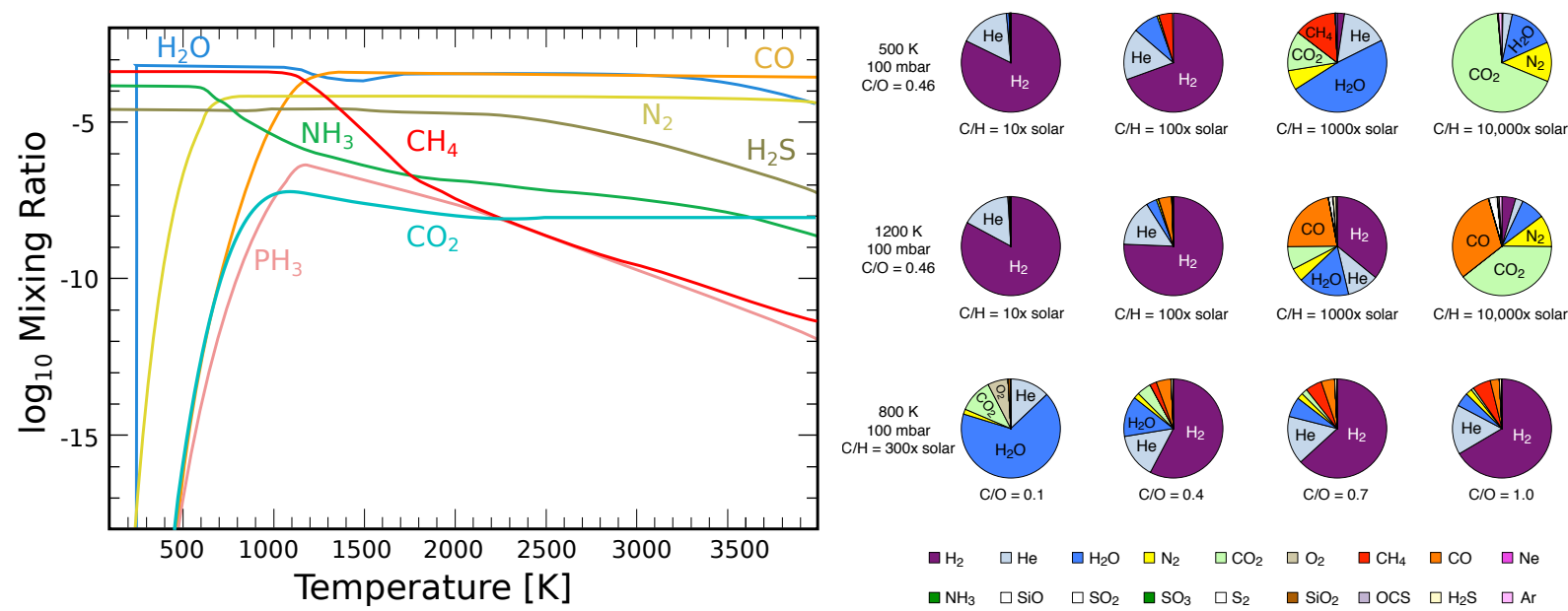

FIG. 4.- Molecular equilibrium abundances: Left: vs. temperature in a Solar-metallicity atmosphere at a total pressure of 1 bar; rainout, photochemistry, quenching, etc. are all neglected (adapted from Sharp \& Burrows 2007. Miguel \& Kaltenegger 2014). $\mathrm{H}_{2}$ would be above the top of the plot. Right: For generic hot Neptunes with a range of temperatures, metallicities, and C/O ratios (from Moses et al. 2013).

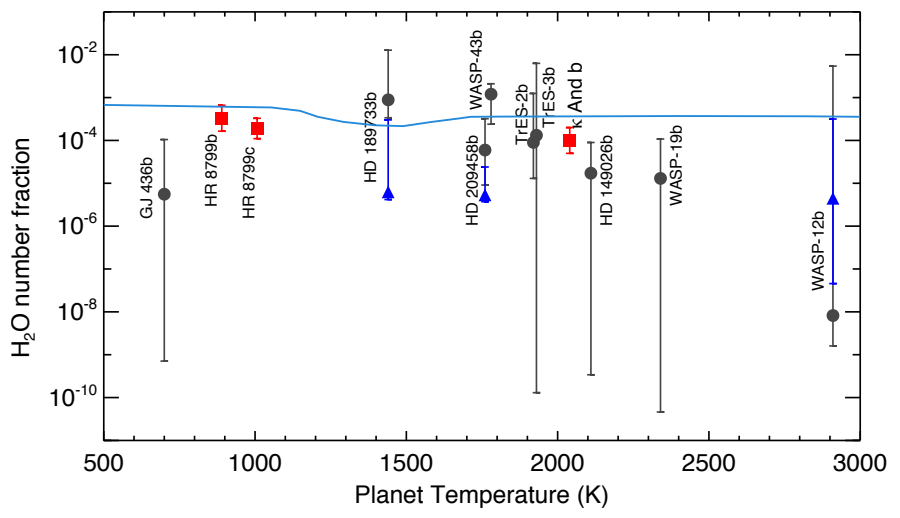

FIG. 5.- $\mathrm{H}_{2} \mathrm{O}$ abundances reported in extrasolar planets. Red squares indicate directly imaged planets, while the grey circles and blue triangles indicate transiting planet emission and transmission spectra, respectively. No clear trends with temperature (or planet mass) are apparent in the present data. Figure adapted from Todorov et al. (2015), with recent data added (Diamond-Lowe et al. 2014 Barman et al. 2015

dances are at least roughly consistent with equilibrium conditions and a Solar-metallicity atmosphere (Todorov et al. 2015). As with $\mathrm{CO}, \mathrm{H}_{2} \mathrm{O}$ abundance measurements of exoplanets should considerably advance in the coming years: with spectra of directly imaged planets from GPI and SPHERE, and by virtue of JWST's higher sensitivity and broader wavelength coverage.

$\mathbf{C H}_{4}$ is expected to be less abundant than $\mathrm{CO}$ in all but the coolest exoplanets studied to date (see Fig. 4 ). Furthermore, even in cooler planets disequilibrium processes tend to further decrease the $\mathrm{CH}_{4}$ abundance in favor of CO (see Sec. 4.3). The most convincing spectroscopic detection of $\mathrm{CH}_{4}$ to date is for the directly-imaged planet HR $8799 \mathrm{~b}$, which exhibits a $\mathrm{CH}_{4}$ abundance consistent with expectations from a Solar-like metallicity and typical disequilibrium conditions (see Fig. 3). No conclusive, spectroscopically resolved measurements of $\mathrm{CH}_{4}$ have been made in transiting planets, despite numerous attempts (Swain et al. 2008, Gibson et al. 2011; de Kok et al. 2013; Birkby et al. 2013). A peculiar emission feature repeatedly and consistently detected from HD $189733 \mathrm{~b}$ at $3.3 \mu \mathrm{m}$ has been attributed to $\mathrm{CH}_{4}$ (Swain et al. 2010b; Waldmann et al. 2012), but this detection is contested (see Sec. 4.3). HST/WFC3 spectroscopy has the sensitivity and wavelength coverage necessary to probe for $\mathrm{CH}_{4}$ in cool transiting planets (Benneke \& Morley, in prep), but no detections have been made so far.

Other molecules: At temperatures $\lesssim 500 \mathrm{~K}, \mathbf{N H}_{3}$ becomes increasingly abundant (see Fig. 4). This molecule has been detected in sub-equilibrium abundances in cool brown dwarfs and solivagant (isolated, free-floating) planetary-mass objects (Leggett et al. 2013. Line et al. 2014a, 2015), but has not yet been seen in any exoplanetary atmosphere. At the warmer temperatures of hot Jupiters, TiO and VO could cause prominent features similar to those seen in M stars (Reid \& Hawley 2005). Tentative spectroscopic evidence for TiO was reported in HD 209458b (Désert et al. 2008), but subsequent high-dispersion spectroscopy shows no evidence of the molecule (Hoeijmakers et al. 2015). Recent eclipse observations of WASP-33b led to a claimed detection of TiO (Haynes et al. 2015), but as the data show large systematics and neither alternative optical absorbers nor reflection were considered in the analysis, the detection should be regarded as tentative.

\subsubsection{Molecular Abundances from Broadband Photometry}

Even when individual lines or bands are not resolved, a single bandpass well-centered on a particular absorption or emission feature can in principle constrain the abundance of the species of interest. However, the data must be of high quality: while systematic noise is unlikely to mimic the strengths and spacing of a high-dispersion $\mathrm{CO}$ spectrum, correlated errors can more easily bias a single broadband measurement. Broadband measurements obtained at multiple epochs are more susceptible to calibration drifts than is spectroscopy, for example when comparing transit measurements when the host star is variable and/or spotted (e.g., Knutson et al. 2011; Pont et al. 2013, Barstow et al. 2015). Despite these challenges, broadband data are almost always easier to acquire than is spectroscopy, so photometry has historically 
preceded any dedicated spectroscopic followup.

The Spitzer/IRAC camera has observed photometric transits and eclipses of more planets than any other facility, and has often been employed in conjunction with ground-based imagers. Though early Spitzer photometry was used to claim various molecular detections and atmospheric features (see Sec. 6.4), subsequent analyses significantly revised or cast doubt on many of the past measurements (Diamond-Lowe et al. 2014; Zellem et al. 2014b; Lanotte et al. [2014; Evans et al. [2015; Morello et al. 2015) and led to the suggestion that broadband transit photometry may be substantially less precise than previously claimed (Hansen et al. 2014). In light of these revisions it is debatable whether broadband photometry usefully determines atmospheric abundances in any transiting exoplanets. Transit or eclipse photometry with JWST may suffer from similar ambiguities, so plans to use such techniques to, e.g., study habitable super-Earths' atmospheres should be treated with great caution (Kaltenegger \& Traub 2009, Deming et al. 2009).

Perhaps the most robust Spitzer result is the thermal emission spectrum of GJ 436b, a transiting, $\sim 700 \mathrm{~K}$, Neptune-mass planet. Though reported secondary eclipse depths differ by $>4 \sigma$ in some bandpasses, analyses agree that planetary emission is stronger in the $3.6 \mu \mathrm{m}$ band but weaker at $4.5 \mu \mathrm{m}$ (Stevenson et al. 2010, 2012. Beaulieu et al. 2011; Lanotte et al. 2014). Since the strongest absorbers are expected to be $\mathrm{CH}_{4}$ at $3.6 \mu \mathrm{m}$ and $\mathrm{CO}_{2}$ and $\mathrm{CO}$ at $4.5 \mu \mathrm{m}$, numerous independent analyses conclude that GJ $436 \mathrm{~b}$ is enriched in $\mathrm{CO}$ and $\mathrm{CO}_{2}$ and depleted in $\mathrm{CH}_{4}$ compared to equilibrium expectations for a Solar-abundance atmosphere (Madhusudhan et al. 2011b, Line et al. 2013, Moses et al. 2013). The interpretation of these analyses is discussed in Sec. 4.3 .

Photometry seems somewhat more reliable for directly imaged planets. The standard ground-based nearinfrared bandpasses sample the spectral energy distributions of a large number of these systems, but to constrain atmospheric chemistry — especially the relative abundances of carbon-bearing species — observations at longer wavelengths $(\gtrsim 3 \mu \mathrm{m})$ are optimal. Groundbased photometry at $3-5 \mu \mathrm{m}$ has probed the atmospheres of several young, directly imaged giant planets (e.g., Skemer et al. 2014a), implying $\mathrm{CO} / \mathrm{CH}_{4}$ ratios up to $100 \times$ greater than expected in equilibrium (see Sec. 4.3. Broadband observations have also led to inferences about cloud properties in these planets, as discussed in Sec. 5 .

Perhaps the most exciting ground-based photometric measurements to date are of the 160 Myr-old GJ 504b (Kuzuhara et al. 2013). Photometry on and off of the $1.6 \mu \mathrm{m} \mathrm{CH}_{4}$ absorption band shows strong emission outside the band and no detection within, indicating strong $\mathrm{CH}_{4}$ absorption (Janson et al. 2013, Skemer et al., in prep.) and perhaps the first robust detection of $\mathrm{CH}_{4}$ in any exoplanet atmosphere. With $T_{\text {eff }} \lesssim 600 \mathrm{~K}$, GJ $504 \mathrm{~b}$ is the coolest exoplanet whose thermal emission spectrum has been studied. This detection of $\mathrm{CH}_{4}$ suggests that fundamentally new classes of directly imaged planets are about to be studied; rapid progress will likely be made in the coming years via followup studies of this and other similarly cool planets with GPI, SPHERE, and JWST.

\subsection{Carbon-to-Oxygen Ratios}

Carbon and Oxygen are the two most common elements in the Sun after H and He (Asplund et al. 2009). These two elements, so critical for life on Earth, are expected to predominately form just a few dominant molecular species in the planetary atmosphere studied to date when in chemical equilibrium. $\mathrm{CO}, \mathrm{H}_{2} \mathrm{O}, \mathrm{CO}_{2}$, and $\mathrm{CH}_{4}$ should all induce prominent spectral features when present in sufficient abundances. A gas or ice giant's bulk composition — and perhaps its atmospheric makeup - should be determined by the location in the disk where the planet accretes most of its gas envelope (Öberg et al. 2011). In particular, the carbon to oxygen (C/O) ratio of a planet's atmosphere will strongly affect the relative abundances of these different molecules (see Fig. 4. also Madhusudhan 2012, Heng et al. 2015, Heng \& Lyons, submitted) and may hold clues to the planet's formation and evolution (van Boekel et al., in prep.)

To date, there is at best tentative evidence for any exoplanet with a $\mathrm{C} / \mathrm{O}$ ratio significantly different from that of its host star and no evidence for $\mathrm{C} / \mathrm{O}>1$. Mediumresolution spectroscopy of the directly-imaged planets HR 8977b and $\mathrm{c}$ show their atmospheres to be consistent with roughly solar $\mathrm{C} / \mathrm{O}$ of $\sim 0.65 \pm 0.1$ (Konopacky et al. 2013. Barman et al. 2015), and high-dispersion spectroscopy of the non-transiting hot Jupiter HR 179949b revealed a low-S/N measurement of $\mathrm{C} / \mathrm{O}=0.5_{-0.4}^{+0.6}$ (Brogi et al. 2014). These spectroscopic measurements use data that directly resolve spectral features from each particular C- or O-bearing molecule; they therefore represent the current "gold standard" for these type of measurements.

Observations of transiting gas giants, typically relying on broadband photometry but with a growing number of spectroscopic data sets, also find no unusual $\mathrm{C} / \mathrm{O}$ ratios. A uniform analysis of nine hot, giant transiting planets showed no evidence for $\mathrm{C} / \mathrm{O}>1$ (Line et al. 2014b). A similar analysis of six additional hot Jupiters is inconclusive: it showed that some broadband measurements were better fit by models with $\mathrm{C} / \mathrm{O}>1$, but the final interpretation is unclear since no uncertainties were reported for the derived planetary $\mathrm{C} / \mathrm{O}$ values (Madhusudhan 2012). A third, independent analysis of data from six hot Jupiters again showed no evidence for $\mathrm{C} / \mathrm{O}>1$ (Benneke 2015).

The most controversial $\mathrm{C} / \mathrm{O}$ measurements have been for the hot Jupiter WASP-12b. On the basis of broadband photometry, this bloated hot Jupiter was the first planet claimed to have $\mathrm{C} / \mathrm{O}>1$ (Madhusudhan et al. 2011b). Additional eclipse measurements (Cowan et al. 2012 ; Crossfield et al. 2012a, Swain et al. 2013), accounting for a nearby $\mathrm{M}$ dwarf binary (Crossfield et al. 2012a Bechter et al. 2014), and an independent retrieval analysis (Line et al. 2014b) demonstrated that on the contrary, existing data were insufficient to justify claims of $\mathrm{C} / \mathrm{O}>1$. New measurements and a reanalysis of old data, and atmospheric retrieval including additional molecules (e.g., $\mathrm{HCN}$ ) generated a counter-claim that WASP-12b does show $\mathrm{C} / \mathrm{O}>1$ (though this last analysis ignored multiple data points not well-matched by the data; Stevenson et al. 2014a). Most recently, new HST/WFC3 data again point to a roughly Solar C/O ratio, leaving no unusually carbon-rich planets in the field (Kreidberg et al. 2015, Benneke 2015). 
High-S/N spectroscopy of directly-imaged planets seems the best near-term hope for additional, more precise measurements of $\mathrm{C} / \mathrm{O}$ ratios. GPI, SPHERE, and similar instruments should enable these measurements for a growing sample of planets and measure their atmospheric abundances. In the longer term, JWST spectroscopy of both transiting and directly imaged planets will offer broader wavelength coverage than Spitzer or HST and significantly improve $\mathrm{C} / \mathrm{O}$ constraints for transiting planets as well.

\subsection{Disequilibrium Chemistry and High Metallicity Atmospheres}

Within the Solar System, no atmosphere is in chemical equilibrium and all exhibit higher metallicity than the Sun. At high altitudes photochemistry caused by the Sun's irradiation induces new reactions, such as the formation of $\mathrm{O}_{3}$ (ozone) from $\mathrm{O}_{2}$ in the Earth's upper atmosphere (Chapman 1930). Vigorous internal mixing deeper in the atmosphere can "quench" abundances at higher altitudes, and is responsible for the CO observed in Jupiter's cold atmosphere (Prinn \& Barshay 1977). As for metallicity, the Solar System's gas giants become increasingly enriched in heavier elements with decreasing mass, with Jupiter exhibiting $\sim 3 \times$ Solar abundances and the ice giants $\gtrsim 50 \times$ Atreya \& Wong 2005 . Karkoschka \& Tomasko 2011); the local terrestrial planets have negligible $\mathrm{H}_{2}$ and so are almost entirely 'metallic' atmospheres.

Given the important role that disequilibrium and elemental composition play in shaping local planetary atmospheres, it is logical that they should influence exoplanetary atmospheres as well. At the high temperatures of the hottest hot Jupiters, kinetic reactions occur so rapidly that all species are driven toward equilibrium abundances. Disequilibrium processes should most strongly affect the atmospheres of planets with $T \lesssim 2000 \mathrm{~K}$ (e.g., Moses et al. 2011; Miguel \& Kaltenegger 2014), which includes all directly imaged planets and most of the transiting planets. Line \& Yung (2013) presents the modified equilibrium constant as a metric for assessing the presence of disequilibrium conditions in a hot exoplanet's atmosphere: $\alpha=\left(f_{\mathrm{CH}_{4}} f_{\mathrm{H}_{2} \mathrm{O}}\right) /\left(f_{\mathrm{CO}} f_{\mathrm{H}_{2}}^{3} P^{2}\right)$, where the $f_{i}$ are the mixing ratios of the several species, $P$ is the atmospheric pressure level probed by observations, and $\alpha$ is to be compared with the value expected in equilibrium (see Heng et al. 2015 for an elegant derivation of this relation and discussion of disequilibrium conditions). Current uncertainties on molecular abundances are large; nonetheless Fig. 6 shows $\alpha$ for exoplanet atmospheres with useful abundance constraints. Cooler planets like GJ 436b and HR 8799b may exhibit disequilibrium conditions, whereas planets hotter than $\sim 1200 \mathrm{~K}$ are near equilibrium. The $3000 \mathrm{~K}$ WASP-33b is a possible hightemperature outlier (Haynes et al.2015), warranting further studies of this object.

\subsubsection{In Transiting Planets}

Most discussions of disequilibrium chemistry and high metallicity in transiting exoplanet atmospheres relate to the thermal emission spectrum of the $\sim 700 \mathrm{~K}$ hot Neptune GJ 436b, which is the current best candidate for exhibiting strong disequilibrium effects (Line \& Yung

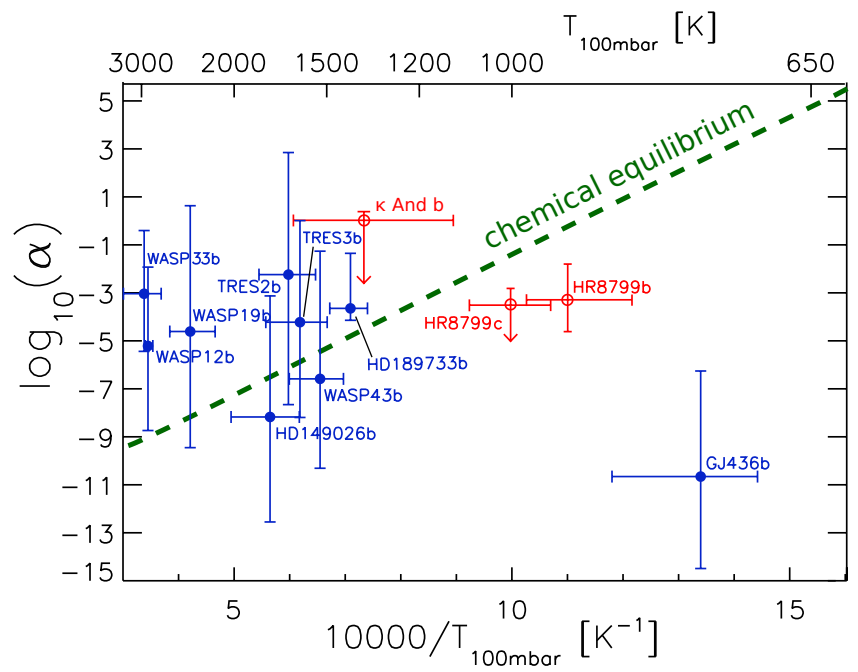

Fig. 6.- Evidence for disequilibrium chemistry in exoplanetary atmospheres. In equilibrium $\alpha$ should increase with decreasing $T$, as indicated by the dashed line. Transiting plants are in blue, directly imaged planets are in red. Photospheric abundances in hotter planets are typically consistent with chemical equilibrium, but disequilibrium effects become increasingly apparent below $\sim 1200 \mathrm{~K}$. Adapted from Line \& Yung (2013) and updated with recent data (Konopacky et al. 2013 Todorov et al. 2015, Barman et al. 2015 Haynes et al. 2015).

2013). Fig. 4 shows that in an equilibrium, Solarmetallicity atmosphere at this temperature $\mathrm{CH}_{4}$ should be much less abundant than CO. Yet as described in Sec. 4.1.2, although the shape of the planet's spectrum is still under debate multiple analyses agree that the planet emits at $3.6 \mu \mathrm{m}$ (where $\mathrm{CH}_{4}$ would absorb) but shows no emission at $4.5 \mu \mathrm{m}$ (where $\mathrm{CO}$ and/or $\mathrm{CO}_{2}$ would absorb).

While photochemistry alone cannot destroy enough $\mathrm{CH}_{4}$ to explain GJ 436b's spectrum, the combination of photochemical $\mathrm{CH}_{4}$ destruction and vigorous internal eddy diffusion to quench high-altitude $\mathrm{CH}_{4}$ and $\mathrm{CO}$ could explain the observed spectrum (Line et al. 2011, Madhusudhan \& Seager 2011; Moses et al. 2013). AIternatively, the high $\mathrm{CO} / \mathrm{CH}_{4}$ ratio can be fit with an extremely high-metallicity atmosphere, with an enhancement $\geq 300 \times$ over Solar (Moses et al. 2013, Fortney et al. 2013). Such an atmosphere might result if only a modest $\mathrm{H}_{2}$ envelope were accreted by the young planet; the low $\mathrm{H}$ abundance naturally leads to a preference for $\mathrm{H}$-poor molecules like $\mathrm{CO}$ over $\mathrm{H}$-rich molecules like $\mathrm{CH}_{4}$. An interesting twist on the $\mathrm{H}_{2}$-poor atmosphere hypothesis would be a He-dominated atmosphere, left over after near-complete erosion of a primordial $\mathrm{H}_{2}$ envelope ( $\mathrm{Hu}$ et al. 2015). The current data quality for GJ $436 \mathrm{~b}$ is too poor to discriminate between all these theories, but JWST eclipse spectroscopy should demonstrate the extent to which disequilibrium, highmetallicity, and unusual abundance patterns affect the atmosphere of GJ 436b and other sub-Jovian planets.

A possible but controversial indication of disequilibrium processes comes from the hot Jupiter HD 189733b. Ground-based spectroscopic observations of this planet's thermal emission show unexpectedly strong thermal emission at $3.3 \mu \mathrm{m}$ (Swain et al. 2010b; Waldmann et al. 2012), attributed to $\mathrm{CH}_{4}$ fluorescence analogous to that seen on Jupiter, Saturn, and Titan (Drossart et al. 1999 
Kim et al. 2000). The feature could potentially be produced by incorrect data calibration (Mandell et al. 2011; Crossfield et al. 2012b), but the consistent detection of this spectral feature from HD 189733b on multiple nights and the absence of any such feature in otherwise identical observations of HD 209458b (Zellem et al. 2014a) suggests that the detection may be genuine. Eclipse spectroscopy of HD 189733 b with JWST will easily detect any such feature, determine whether it is caused by $\mathrm{CH}_{4}$, and so settle the matter.

\subsubsection{In Directly Imaged Planets}

The higher-quality data available for directly imaged planets allows more detailed studies of atmospheric chemistry and disequilibrium than is possible for transiting systems. Spectroscopy of the accessible planets reveals that they, as for the transiting GJ 436b described above, exhibit $\mathrm{CO} / \mathrm{CH}_{4}$ ratios significantly greater than would be expected from equilibrium models and Solar abundances (Barman et al. 2011a b, 2015; Lee et al. 2013 Skemer et al. 2014a). Stellar irradiation and photochemistry is negligible for these young, hot planets, so vigorous internal eddy diffusion is presumably responsible for quenching the upper-atmospheric abundances at the equilibrium values lower in the planets' atmospheres. Curiously, despite the high-quality data available for directly imaged planets molecular abundances for these systems are less frequently reported than for transiting planets. With spectroscopy of many additional systems expected soon from high-contrast spectrographs, the field would benefit from a more systematic analysis of these planets' atmospheric conditions and molecular abundances (see Sec. 3).

\subsection{Alkalis, Ions, and Exospheres}

Highly irradiated planets such as hot Jupiters have temperatures on their day sides up to roughly $3000 \mathrm{~K}$ - as hot as some M stars - at pressures of $\sim 1$ bar. At lower pressures (higher altitudes), the atmospheric density steadily decreases until the conditions for local thermodynamic equilibrium are no longer met. In the high-altitude exosphere, temperatures can reach up to $\sim 10,000 \mathrm{~K}$, hot enough to split molecules and partially ionize the constituent atoms. Fig. 7 shows theoretical abundance profiles of some of the more common species predicted to exist in these planets' atmospheres (Lavvas et al. 2014). In some cases the abundances are predicted to be even greater than shown here as a planet's $\mathrm{H}_{2}$-dominated atmosphere escapes to space and heavier species are entrained in the resulting outflow. The studies of these several phenomena are summarized in the recent review by Fossati et al. (2015).

Alkali species such as sodium were the first constituents detected in a hot Jupiter's atmosphere (Charbonneau et al. 2002, Redfield et al. 2008). Mass loss and high-altitude atomic species have been observed in a growing number of short-period transiting planets using ultraviolet HST transit spectroscopy. These detections include increased absorption in $\mathrm{H} \alpha$ (e.g., Vidal-Madjar et al. 2003), oxygen (Vidal-Madjar et al. 2004), carbon and silicon (Linsky et al. 2010), and tentative detections in other high-order ultraviolet metal lines (Fossati et al. 2010, Haswell et al. 2012).
Mass loss has been observed for a number of hot Jupiters through the detection of extremely deep transits in excitation lines of hydrogen and various metals, especially via ultraviolet transit spectroscopy with HST (Vidal-Madjar et al. 2003, 2004, Linsky et al. 2010; Fossati et al. 2010). Qualitatively, the mass loss is understood to result from hydrodynamic Roche lobe overflow of the planet's exosphere, powered by the extremely highenergy X-ray and FUV flux of the host star. However, quantitative models of this phenomenon produce mass loss rates and conditions in the exosphere which vary by several orders of magnitude (Penz et al. 2008, MurrayClay et al. 2009 Koskinen et al. 2013) indicating that the detailed mechanisms involved remain only poorly understood.

Atmospheric mass loss from lower-mass planets is even less well studied than from hot Jupiters; the only observation to date are Ly $\alpha$ transit observations of hot Neptune GJ $436 \mathrm{~b}$ and the $\sim 2000 \mathrm{~K}$ sub-Neptune $55 \mathrm{Cnc}$ e. The nondetection of Ly $\alpha$ around the latter sets an upper limit to the planet's hydrogen mass-loss rate of $\sim 3 \times 10^{8} \mathrm{~g} \mathrm{~s}^{-1}$ (Ehrenreich et al. 2012). More exciting is the substantial hydrogen envelope recently detected around GJ 436b, seen as a $\sim 50 \%$ transit depth in Ly $\alpha$ and indicating a mass-loss rate of $\sim 10^{8}-10^{9} \mathrm{~g} \mathrm{~s}^{-1}$ (Ehrenreich et al. 2015). The upper range of both these measurements are a factor of a few lower than those predicted by Lammer et al. (2013), and 10-100 times lower loss rates predicted by other studies (Valencia et al. 2010. Owen \& Jackson 2012).

\section{CLOUDS \& HAZES}

As with disequilibrium chemistry (see Sec. 4.3), clouds and hazes are ubiquitous in all Solar system planets with thick atmospheres. A growing body of evidence indicates that atmospheric condensates are also common in extrasolar planet atmospheres. Clouds form when gaseous species condense out of the atmosphere, forming liquid droplets or solid 'dust' particles. Fig. 8 shows condensation curves for compounds that may be present in exoplanetary atmospheres. These clouds may form at points where the condensation curves intersect a planet's atmospheric thermal profile. It is clear from Fig. 8 that a wide diversity of clouds are possible. The section below first discusses the early signs of clouds in brown dwarfs and more recent signs in their less-massive, directly imaged cousins, and follows with the evidence for clouds and haze in transiting exoplanets. Interested readers are strongly recommended to read the in-depth review by Marley et al. (2013), which gives a much more detailed description of clouds and hazes in both exoplanets and brown dwarfs.

\subsection{Brown Dwarfs and Young, Massive Planets}

As brown dwarfs age and slowly cool, clouds of condensed refractory compounds (e.g., calcium aluminates, silicates, and iron) start to form in their atmosphere (Lunine et al. 1986, Lodders 1999). As the cooling continues, brown dwarfs transition from dusty atmospheres (Kirkpatrick et al. 1999, Martín et al. 1999) to dust-free conditions (Burgasser 2001; Burgasser et al. 2002) when the effective temperature reaches $\sim 1300 \mathrm{~K}$ (Stephens et al. 2009, Testi 2009). Different models have been proposed to explain this dust dispersal and include either a sudden 


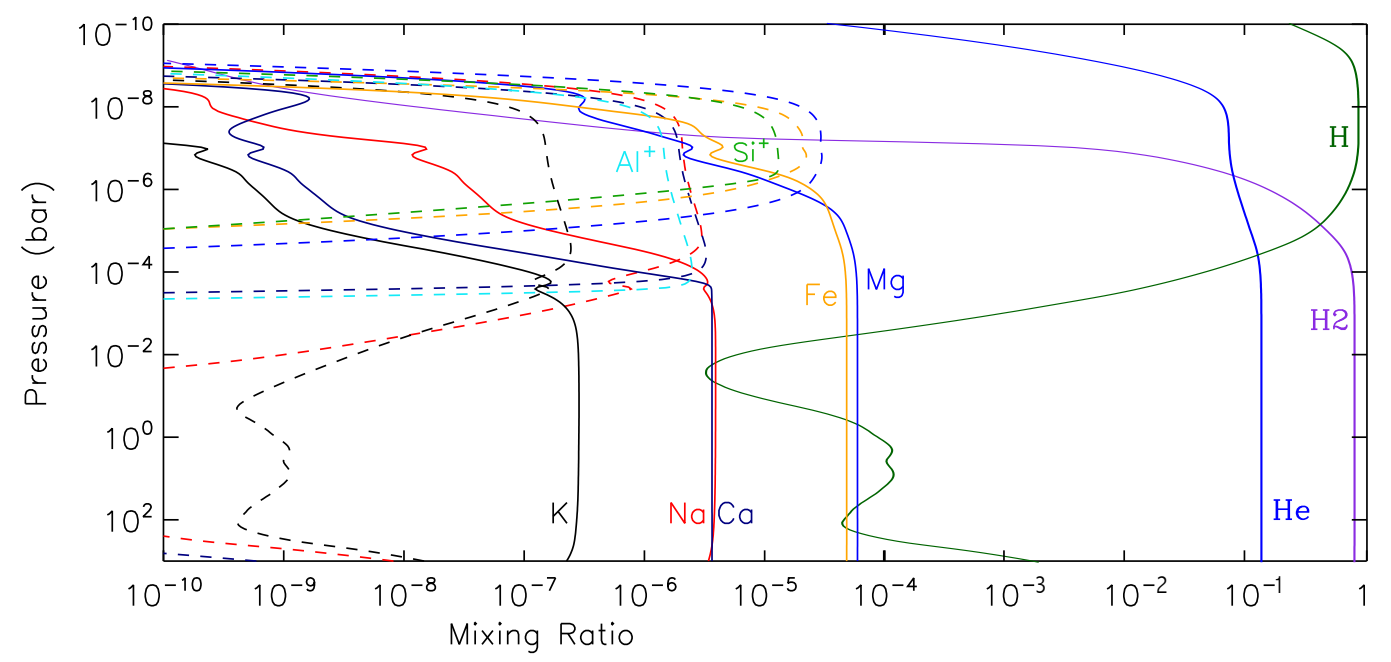

FIG. 7. - Theoretical abundance profiles of atomic and ionized species in the atmosphere of hot Jupiter HD 209458b. Solid lines are neutral species; dashed lines of the same color indicate the corresponding ion. Observations of these species probe mbar-to- $\mu$ bar levels, much higher than the bar-to-mbar levels probed by thermal emission measurements. Adapted from Lavvas et al. (2014).

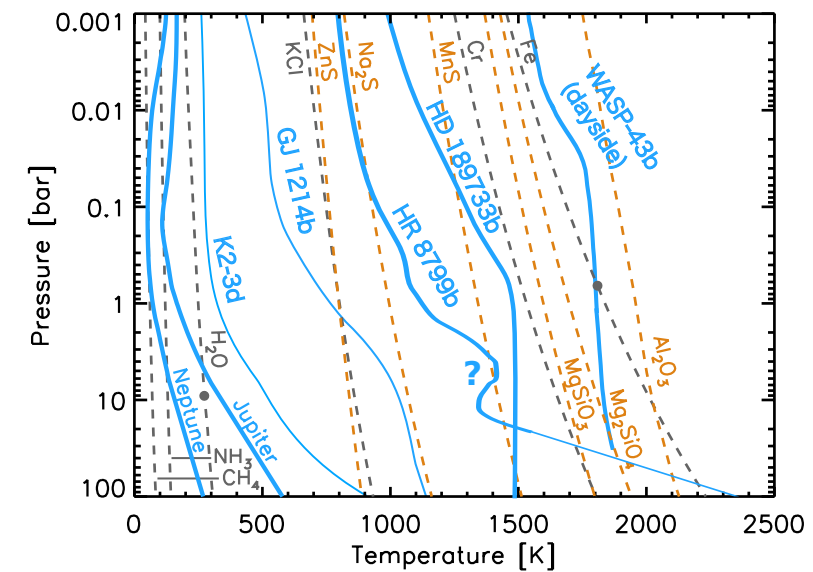

FIG. 8.- Condensation curves from Marley \& Robinson (2014) for various refractory species assuming Solar metallicity (dashed lines) compared to temperature-pressure profiles of known planets (solid lines). Thick lines are empirical measurements from atmospheric retrieval analyses (Lee et al. 2013 Crouzet et al. 2014 Stevenson et al. 2014b); thin lines are theoretical models (Crosshield et al. 2011 T. Barman et al., private communication). The retrieved exoplanet profiles are all essentially unconstrained at $\gtrsim 3$ bar and $\lesssim 10$ mbar. Thus the kink in HR 8799b's profile could be either a numerical artifact or the result of a thick, low-altitude cloud layer.

clearing or collapse of the cloud (Stephens et al. 2009, Allard et al. 2003; Knapp et al. 2004; Tsuji \& Nakajima 2003 Tsuji et al. 2004) or patchy regions of varying cloud thickness (Burgasser et al. 2002; Ackerman \& Marley 2001; Marley et al. 2010) as seen on Jupiter and Saturn. To the extent that brown dwarfs are just more massive versions of young, directly imaged planets, the same trends should hold. However, the lower gravity of planets means that clouds remain in the visible photosphere for much fainter planets than for brown dwarfs (e.g., Barman et al. 2011a). Broadband photometry of many directly imaged planets confirms that clouds are required to match atmospheric models to observations, even when the planet is substantially cooler than cloudfree brown dwarfs (Madhusudhan et al. 2011a: Currie et al.|2011; Skemer et al.2014a). The near-infrared colors of the cooler, fainter GJ 504b suggests that the transition to more nearly cloud-free conditions occurs at lower temperatures than in brown dwarfs (Kuzuhara et al. 2013).

$\mathrm{Y}$ dwarfs are objects with $T_{\text {eff }} \lesssim 450 \mathrm{~K}$ and low masses $\left(5-30 M_{J u p}\right)$ that approach or straddle the canonical planetary-mass boundary (Cushing et al. 2011, Kirkpatrick et al. |2012; Leggett et al. 2013 Dupuy \& Kraus 2013 Beichman et al. 2014b). In addition to substantial ammonia $\left(\mathrm{NH}_{3}\right)$, their atmospheres contain many types of cloud species. In the warmest $\mathrm{Y}$ dwarfs these clouds should be composed of $\mathrm{Na}_{2} \mathrm{~S}$, and indeed warmer $\mathrm{Y}$ dwarfs are better fit by atmospheric models including clouds than by cloud-free models (Morley et al. 2012 Beichman et al. 2014b). At temperature $\lesssim 300 \mathrm{~K}$ the condensed species may include $\mathrm{H}_{2} \mathrm{O}, \mathrm{NH}_{3}$, and other, more exotic species (Burrows et al. 2003, Visscher et al. 2006: Morley et al. 2014). No models match the existing broadband data for the coolest objects $(\lesssim 250 \mathrm{~K}$; Luhman 2014; Kopytova et al. 2014), emphasizing that our understanding of these cool, cloudy planetary-mass objects are still quite uncertain.

\subsection{Transiting Planets}

From the earliest models of transiting exoplanet atmospheres (Seager \& Sasselov 2000; Sudarsky et al. 2000; Brown et al. 2001; Hubbard et al. 2001) it was clear that clouds should have a major impact on these planets' observable properties. The effect on transmission measurements can be especially severe. The transit geometry increases the optical depth of absorbers $\gtrsim 50 \times$ over that in the normal, thermal emission geometry, which means that even optically thin species can obscure the atmospheric composition (Fortney 2005).

The most robust evidence of condensate particles in a transiting exoplanet's atmosphere comes from spectroscopy of the $\sim 600 \mathrm{~K}, 2.6 R_{\oplus}$ sub-Neptune GJ 1214b. A steady stream of transit spectroscopy, capped by the high-precision HST/WFC3 spectroscopy shown in Fig. 3. demonstrates that GJ 1214b has an exquisitely flat transmission spectrum (Bean et al. 2010, 2011, Crossfield et al. 2011; Berta et al. 2012; de Mooij et al. 2012; Kreidberg et al. 2014b). The measurements rule out cloud-free at- 
mospheres with absorption from most spectroscopically active species (see Fig. 3), so a high-altitude cloud deck at $P \lesssim 0.1$ mbar seems the most likely interpretation. The aerosol's composition is unknown, but theories include either condensate clouds of $\mathrm{ZnS}$ or $\mathrm{KCl}$ (see Fig. 8 , Morley et al. 2012) or photochemically-produced hydrocarbon hazes (Miller-Ricci Kempton \& Rauscher 2012). A haze at such high altitude obscures all but the strongest absorption features; Barstow et al. (2013) suggest that transit spectroscopy with JWST may be the best hope to descry features from either $\mathrm{CH}_{4}$ (at $3.3 \mu \mathrm{m}$ and $7.5 \mu \mathrm{m}$ ) and/or $\mathrm{CO}_{2}$ (at $4.3 \mu \mathrm{m}$ and $15 \mu \mathrm{m}$ ).

Several other sub-Jovian transiting planets also show fairly flat transmission spectra, but in these cases clouds are required only if the planets have $\mathrm{H}_{2}$-dominated, lowmetallicity atmospheres (cf. Sec. 4.3. Knutson et al. 2014a b; Fraine et al. 2014; Biddle et al. 2014; Ehrenreich et al. 2014). Along with GJ 1214b, all these targets are small $\left(R<5 R_{\oplus}\right)$ and cool $\left(T_{e q} \lesssim 900 \mathrm{~K}\right)$, which may provide a clue to the nature of the obscuring material (Howe \& Burrows 2012; Miller-Ricci Kempton et al. 2012 Morley et al. 2013).

Larger, usually hotter transiting planets appear to be qualitatively different. Most show $\mathrm{H}_{2} \mathrm{O}$ absorption in HST/WFC3 spectroscopy, albeit weaker than expected (for a recent summary see Benneke 2015). The interpretation seems to be that some hot Jupiters have a thin cloud or haze layer that weakens (but does not entirely mask) the signature of $\mathrm{H}_{2} \mathrm{O}$ absorption (e.g., McCullough et al. 2014; Nikolov et al. 2015; Sing et al. 2015). As with the smaller, cooler planets the cloud species and size distribution remains unknown. JWST spectroscopy offers a promising avenue for determining the composition of these planets' clouds. Sub-micron silicate cloud particles could create strong spectral features in these planets' spectra at $\sim 10 \mu \mathrm{m}$ (Lee et al. 2014); nonsilicate materials (e.g., tholins) may show similarly strong features at other mid-infrared wavelengths (Wakeford \& Sing 2015).

Clouds, hazes, or dust have also been suggested for transiting planets showing no conclusive features of any kind in emission (Hansen et al. 2014, Evans et al. 2015), or showing only flat or sloped transmission spectra at optical and infrared wavelengths (Lecavelier Des Etangs et al. 2008b; Sing et al. 2009, Gibson et al. 2013; Sing et al. 2013 Pont et al. 2013). These slopes have been interpreted as the signature of particulate Rayleigh scattering in the upper atmosphere, which conveniently and plausibly accounts for the muted spectral features discussed above and (by absorbing starlight at altitude and so shifting the thermal profile) for the near-blackbody emission spectra of some planets (Crossfield et al. 2012a Knutson et al. 2012; Pont et al. 2013; Burrows 2014b).

However, the interpretation of Rayleigh scattering has been questioned in analyses of HD 189733b and other planets, with the slope instead attributed to a haze-free atmosphere and transit contamination from a nonuniform stellar surface (McCullough et al. 2014, Oshagh et al. 2014). High-precision optical albedo measurements could settle the matter: by constraining the presence and size distribution of any haze particles in these atmospheres (Evans et al. 2013, Barstow et al. 2014). If the particles are $\lesssim 1 \mu \mathrm{m}$, atmospheric circulation models indicate that they should be present throughout the planet's atmosphere (Parmentier et al. 2013) - and in particular, near the terminator probed by transmission spectroscopy.

\section{ATMOSPHERIC CIRCULATION \& ENERGY BUDGETS}

Planetary atmospheres typically exhibit variability and weather patterns on many scales, from terrestrial hurricanes to Jupiter's Great Red Spot to Saturn's dramatic storm of 2010 (Sromovsky et al. 2013, Sayanagi et al. 2013). The atmospheric processes that drive all this global weather (for a recent review see Heng \& Showman 2015) must also play a role in the atmospheres of exoplanets, and so the observed atmospheric properties of extrasolar planets must also be variable at some level. Another wrinkle for short-period planets such as hot Jupiters is that these planets' intense irradiation also leads to severe longitudinal temperature gradients, observed as thermal phase curves. The irradiation drives the planets' circulation and the net input energy is necessarily modulated by the planetary albedo. All these processes combine to determine a planet's thermal structure. The section below discusses each of these phenomena in turn: phase curves in Sec. 6.1, albedos in Sec. 6.2, rotation and variability in Sec. 6.3 and thermal structure (and the lack of any definitive thermal inversions) in Sec. 6.4

\subsection{Phase curves}

To date, phase curves have been measured at different wavelengths, with different cadences, and with varying orbital coverage fractions. Early phase curves of nontransiting hot Jupiters were of limited utility because the then-unknown orbital inclination of our targets introduced degeneracies in the interpretation of these data (Cowan et al. 2007, Crossfield et al. 2010). Now that high-dispersion spectroscopy breaks the $\sin i$ degeneracy (see Sec. 2.4), non-transiting phase curves become more useful. Nonetheless most observations to date focus on transiting systems.

One of the great successes of modeling exoplanet atmospheres was the prediction of super-rotating equatorial winds on hot Jupiters, which leads to hot spots shifted eastward from the substellar point (Showman \& Guillot 2002). This phenomenon is commonly observed in hot Jupiter phase curves, with eastward phase offsets observed for dozens of systems (see Fig. 9p. However, predictions that day/night temperature contrast (and thus phase curve amplitude) should increase with dayside temperature have been disappointed. Some extremely hot Jupiters behave as expected (WASP-12b, WASP-18b), but as shown in Fig. 9 other, much cooler planets also exhibit strong temperature contrasts (e.g., WASP-43b). It is sobering to consider the utility of the data plotted in Fig. 9 if these Spitzer phase curve observations were found to suffer from the same limitations and systematics seen in Spitzer broadband photometry (Sec. 4.1.2).

Current phase curve observations cannot be explained by advection alone, which generically predicts small phase offsets for planets with large temperature contrasts (Fortney et al. 2008; Cowan \& Agol 2011). Fig. 9 shows that this trend is emphatically not observed, which could indicate any combination of dynamically evolving atmospheric chemistry (Agúndez et al. 2012), inhomogeneous clouds (Showman \& Kaspi 2013), or atmospheric drag 

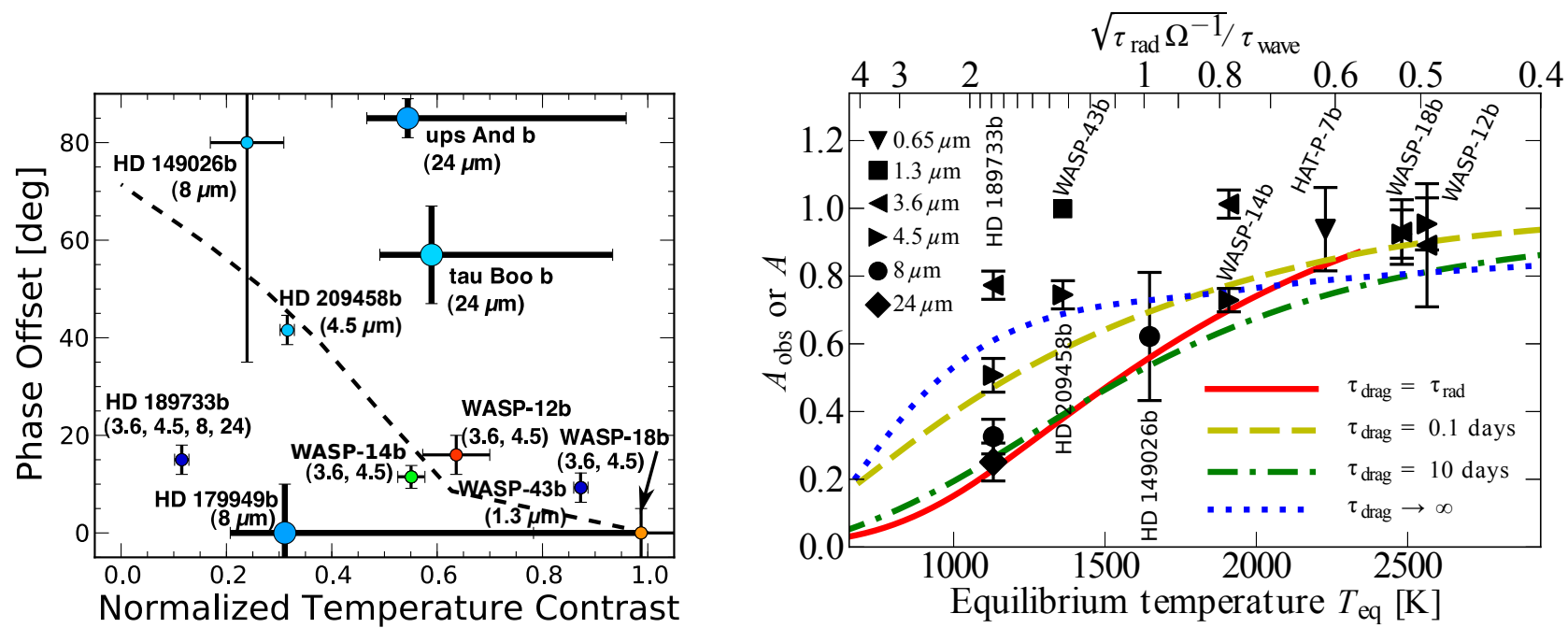

FIG. 9.- : Left: All infrared phase curve observations for hot Jupiters on circular orbits. Temperature contrasts have been normalized by $T_{\text {eq }}$ (indicated by blue and red colors for cooler and hotter planets, respectively). The dashed line is the semi-analytic radiative/advective model of Cowan \& Agol (2011), which predicts smaller phase offsets for larger day/night temperature contrasts. Right: Relative phase curve amplitudes vs. equilibrium temperature, along with shallow-water circulation model predictions for various drag timescales. Figure adapted from Perez-Becker \& Showman (2013), with additional data added (Zellem et al. 2014b Stevenson et al. 2014b Wong et al. 2015).

forces (Rauscher \& Menou 2013, Perez-Becker \& Showman 2013).

Though most phase curves to date have been obtained in one bandpass at a time, recent observations of hot Jupiter WASP-43b reveal the power of phase curve spectroscopy (Stevenson et al. 2014b). This analysis determined the planet's mean $\mathrm{H}_{2} \mathrm{O}$ abundance and thermal structure as a function of longitude, finding a much colder nightside than was expected. Such spectroscopy will likely be used to great effect in the coming years with both HST and JWST.

Phase curves have also been observed from many planets at optical wavelengths, where scattered starlight is at least as important as thermal emission. These analyses often show westward-shifted phase offsets - the reverse of what infrared studies have found. The interpretation has been that reflective clouds form in the planets' cooler regions, reducing thermal emission and boosting reflection (Demory et al. 2013, Shporer \& Hu 2015, Webber et al. 2015 Esteves et al. 2015). While this hypothesis is plausible, caution is advised because the optical modulations could be caused by either scattering in the planet's atmosphere or by star-planet interactions. Optical photometry of the hot Jupiter $\tau$ Boo b revealed optical modulation consistent with a planetary phase curve, but with an amplitude much too large to be of planetary origin (Walker et al. 2008). It seems possible that the more recent detections of optical modulation could be caused by a weaker form of the $\tau$ Boo effect instead of having a solely planetary nature.

\subsection{Albedos}

Broadband photometry of hot Jupiters indicates that they typically have low geometric albedos at optical wavelengths $\left(A_{G} \lesssim 0.1\right.$; e.g., Rowe et al. 2008). In contrast, thermal emission measurements (mostly from Spitzer secondary eclipses) indicate that hot Jupiters' Bond albedos are typically $A_{B} \approx 0.4$ (Schwartz \& Cowan 2015). Fig. 10 demonstrates this curious dichotomy. Most planets studied in this way have been observed with either Kepler alone (and orbit stars too faint for followup with Spitzer or HST) or only with Spitzer (and do not lie in the Kepler field of view). Nonetheless these discrepent families of albedo measurements do not arise from selection effects: HD 189733b and HD 209458b have been observed at both optical and NIR wavelengths, and both show the same discrepancy with $A_{B}>A_{G}$. Similarly, no obvious biases result from the use of Spitzer data since consistent Bond albedos are reported for WASP-43 whether only HST or both HST and Spitzer data are used (Stevenson et al. 2014b; Schwartz \& Cowan 2015). The planets exemplifying this albedo phenomenon span a wide range of temperatures and are unlikely to host the same cloud species, so this issue transcends any single class of planets.

The most likely solution to this issue is that hot Jupiters have substantially higher geometric albedos beyond the optical bandpasses used (Schwartz \& Cowan 2015). Indeed, the first extrasolar albedo spectrum (of HD 189733b; Evans et al. 2013) revealed $A_{G}=0$ within the Kepler bandpass but $A_{G}=0.4$ at $300-$ $450 \mathrm{~nm}$. These observations can be fit by a wide range of atmospheric compositions including any of alkalis, clouds/hazes, or even gaseous $\mathrm{TiO} / \mathrm{VO}$ (Barstow et al. 2014). Such models would also naturally explain the low $A_{G}$ of HD 209458b in the MOST bandpass (Rowe et al. 2008). However, if the estimate of $A_{B} \approx 0.4$ is correct (cf. Fig. 10) then hot Jupiters may also reflect considerable starlight at $\gtrsim 800 \mathrm{~nm}$. Optical eclipse spectroscopy can best test these theories by directly measuring hot Jupiters' geometric albedos as a function of wavelength.

\subsection{Rotation, Variability, \&6 Weather}

Despite the importance of rotation rate, internal heat flux, and external irradiation on planetary weather, these factors are not typically known for extrasolar planets. Short-period giant planets such as hot Jupiters are thought to be tidally locked (Correia \& Laskar 2010). In principle phase curve observations can constrain a planet's rotation rate and test for tidal locking, but 


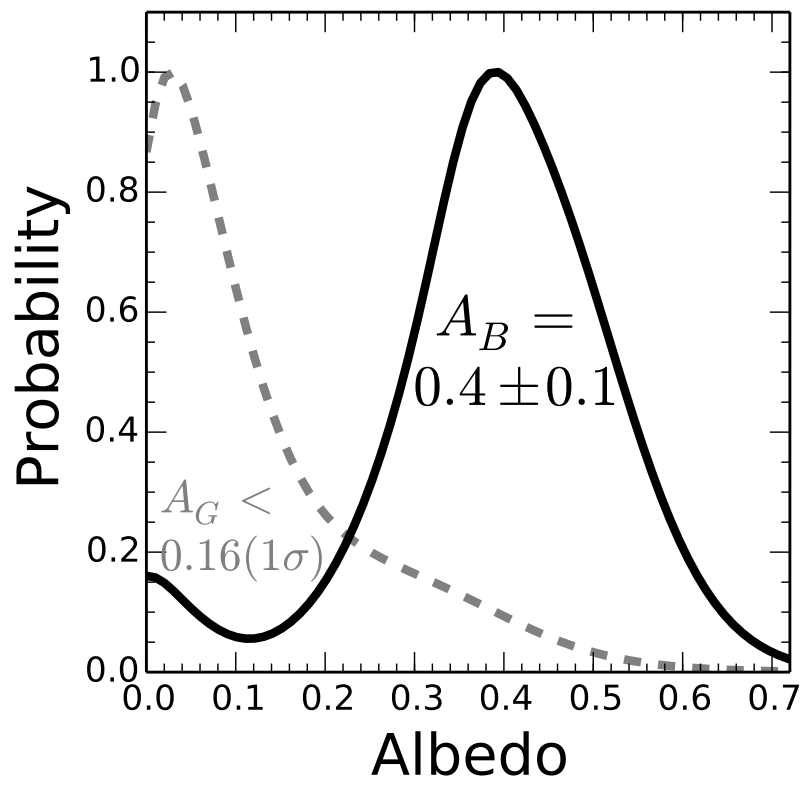

FIG. 10.- Constraints on hot Jupiter albedo and atmospheric recirculation, demonstrating the curious dichotomy between Bond and geometric albedos. Solid lines indicate Bond albedos of planets with infrared flux measurements; these data indicate $A_{B} \approx 0.4$. Dotted outlines indicate geometric albedos of planets with optical secondary eclipses, typically with Kepler; these data indicate $A_{G} \lesssim$ 0.16 in the Kepler bandpass. To explain this striking discrepancy, hot Jupiters must have $A_{G} \gtrsim 0.5$ beyond the Kepler bandpass, at $\lambda<0.4 \mu \mathrm{m}$ and/or $\lambda>0.8 \mu \mathrm{m}$. Data taken from Schwartz \& Cowan (2015) and Wong et al. (2015).

the dependence is highly degenerate with other parameters (e.g., Showman et al. 2009). Lower-mass, shortperiod planets will not necessarily be tidally locked, which will render any interpretation of these planets' phase curves (e.g., with JWST) even more uncertain. High-dispersion spectroscopy can also constrain planetary rotation (Miller-Ricci Kempton \& Rauscher 2012 Kempton et al. 2014 Rauscher \& Kempton 2014), but to date the only observation is a tantalizing hint of windspeeds measured at the terminators of HD 209458b and HD 189733b (Snellen et al. 2010, Wyttenbach et al. 2015).

The best rotation measurement to date came from high-dispersion spectroscopy of the directly imaged planet beta Pic b, which shows a proiected equatorial velocity of $25 \mathrm{~km} \mathrm{~s}^{-1}$ (Snellen et al. 2014). Assuming a radius of $\sim 1.5 R_{J}$ (Bonnefoy et al. 2014b), this rotation period of $\sim 7 \mathrm{hr}$ is shorter than that of Jupiter or Saturn and also less than most brown dwarfs or low-mass stars at a comparable age (Bouvier et al. 2014). The rotation will presumably increase further as the planet ages, cools, and contracts. High-dispersion spectrographs on the next generation of giant telescopes will measure $v \sin i$ for many additional planets, and also create global 2D weather maps via Doppler imaging (Crossfield et al.|2014: Crossfield 2014).

For giant planets that rotate at least as quickly as beta Pic $\mathrm{b}$, detection of rotationally-modulated variability should be feasible in the future. Observations of brown dwarfs reveal rotation rates and variability for many objects (e.g., Artigau et al. 2009; Radigan et al.
2014). Variability seems ubiquitous for brown dwarfs of all spectral types (Buenzli et al. 2014, Metchev et al. 2015), consistent with rotational modulation of an inhomogeneous, cloudy surface as discussed in Sec. 5.1 above. In the case of brown dwarfs, the variability is variously attributed to inhomogeneities in clouds (Apai et al. 2013) or internal thermal perturbations (Robinson \& Marley 2014). High-contrast photometry of directly imaged planets should constrain these same processes in planets rather than brown dwarfs (Kostov \& Apai 2013).

Some early general circulation models of hot Jupiters predicted that even these highly-irradiated planets should not be in a perfectly steady state, but should show substantial inter-epoch variability in thermal emission (Rauscher et al. 2007). Only a handful of these planets have been observed multiple times with the precision necessary to detect variability. The tightest limits come from seven $8 \mu \mathrm{m}$ eclipses of HD 189733b, which ruled out variability above 2.7\% (Agol et al. 2010). Recent Spitzer observations of 55 Cnc e's eclipses suggest that the planet's thermal emission may vary with time, but the underlying cause remains unknown (Demory et al. 2015). Variations in transit depth are more difficult to interpret, since they can much more easily result from starspots and stellar variability rather than from the planet (Agol et al. 2010, Knutson et al. 2011). JWST's enhanced sensitivity ( $\sim 8 \times$ greater than Spitzer's) should allow it to place much tighter constraints on variability of thermal emission, even if transit variability remains dominated by stellar effects.

\subsection{Looking for Thermal inversions}

A planet's thermal structure is said to be inverted when at some altitude its atmosphere satisfies $d T / d P<0$, i.e. when temperature increases with increasing altitude. All of the Solar system's outer, giant planets exhibit temperature inversions above a pressure level of roughly $0.1 \mathrm{bar}$ (Orton 1981), where the outgoing infrared opacity drops and incident radiation begins to dominate (Robinson \& Catling 2014). Such inversions are typically characterized by looking at the planets' thermal spectra, which shows small, narrow emission features in the broader, deeper cores of absorption lines. Isolated brown dwarfs and planetary-mass objects show no such inversions ( $\mathrm{Lu}-$ nine et al. 1986, Stephens et al. 2009, Line et al. 2014a, 2015), but this phenomenon has been long-sought in planets orbiting other stars.

It was appreciated soon after the discovery of hot Jupiters that their intense insolation levels would lead to much shallower T/P profiles (Seager \& Sasselov 1998; Goukenleuque et al. 2000), though it took several more years to realize that short-wavelength absorbers with sufficient optical depth might lead to a strong inversion in these atmospheres (Hubeny et al. 2003). The claimed detection of just such an inversion at $~ 1$ bar in HD 209458b seemed to vindicate these theories (Knutson et al. 2008, but see below) and was used to predict two distinct classes of hot Jupiter atmospheres: those without inversions and those with thermal inversions induced by some high-altitude absorber (Burrows et al. 2008; Fortney et al. 2008). New observations and analyses of other targets seemed to confirm the inversion dichotomy (see Madhusudhan \& Seager 2010: Knutson et al. 2010).

However, it now appears that inversions as initially 
reported are not present in these planets. In an early retrieval analysis of four planets with claimed inversions, data for two planets were equally well fit by models with or without temperature inversions (Madhusudhan \& Seager 2010). A second, similar analysis of nine planets found that all but HD 209458b were best explained by non-inverted atmospheres (Line et al. 2013). A later analysis of new and archival data of HD 209458b then showed that this prototypical inverted atmosphere is not inverted after all (Diamond-Lowe et al. 2014, Zellem et al. 2014b). The best remaining candidates for an inverted atmosphere are HAT-P-7b (Christiansen et al. 2010), which has not been subjected to any reanalysis or atmospheric retrieval; and WASP-33b (Haynes et al. 2015). Even if these last two planets do indeed host thermal inversions, it is clear that the two-class model as originally proposed is now defunct.

\section{THE FUTURE}

As much as any other advances, the considerable recent progress made in characterizing exoplanet atmospheres has come from the development of reliable spectrographic instruments and techniques and from the continued discovery of of new planetary systems that can be more easily observed at high S/N. Progress in both these areas will only accelerate in the coming years: spectroscopy with new facilities such as JWST, WFIRST-AFTA, and ground-based extremely large telescopes, and discoveries of many new planets from ongoing or imminent surveys from GPI, SPHERE, TESS, and GAIA.

\subsection{The Near Term: 2015-2025}

Direct imaging surveys using GPI, SPHERE, and similar instruments are already underway. These will discover and obtain low-resolution spectroscopy of perhaps dozens of young, self-luminous planets. The improved contrast provided by these facilities over existing instruments (see Fig. 2) will also permit higher-quality spectra of planets currently known. The astrometric GAIA mission, also underway, is projected to find tens of thousands of planets on several-AU orbits; a fraction of these will also be accessible to characterization via direct imaging (Perryman et al. 2014, Sozzetti et al. 2014). Spectroscopy of these systems with ground-based systems and with JWST's significantly broader wavelength coverage (Beichman et al.2010) will place ever-tighter constraints on the composition, chemistry, and thermal structure of giant planetary atmospheres. Planetary variability and rotation rates will provide insight into circulation and cloud processes for a growing number of planets. Opticalwavelength observations may reveal signs of ongoing accretion and planet formation (Sallum et al., in prep.), and heroic efforts for a small number of systems could begin to detect starlight reflected from old, cold gas giants. Studies of brown dwarfs will continue to lead the way in demonstrating what can be learned about cool, substellar atmospheres from reliable, high-quality data (e.g., Line et al. 2015).

The upcoming Transiting Exoplanet Survey Satellite (TESS) mission, scheduled for launch in 2017, will find thousands of new terrestrial planets, sub-Jovians, and hot Jupiters transiting bright stars (Ricker et al. 2014). Many of these will be suitable for atmospheric study with JWST via transits, eclipses, and phase curves
(Batalha et al. 2013, Beichman et al. 2014b). Proposals for JWS'T's Guaranteed 'Time and Cycle 1 observations are scheduled for 2017, which means that TESS' planets will arrive too late for observation in the first 1-2 years of JWST operations. Fortunately, the K2 mission (Howell et al. 2014) is already finding planets suitable for JWST transmission spectroscopy (Vanderburg et al. 2015: Crossfield et al. 2015; Montet et al. 2015).

There is an outside chance that transmission spectroscopy with JWST could reveal the features of truly Earthlike atmospheres orbiting late-type M dwarfs (Kaltenegger \& Traub 2009, Deming et al. 2009). Such measurements will be extremely challenging for even relatively favorable planetary systems; whether these efforts will succeed depends on JWST's on-orbit systematic noise floor, which is as yet unknown. Depending on the success of JWST's transit spectroscopy, TESS and K2's discoveries may justify development of dedicated instruments or missions for dedicated spectroscopic surveys of large numbers of these new systems (Swain et al. 2010a; Swain 2012, Glauser et al. 2013, Tinetti et al. 2015; Cowan et al. 2015).

The next generation of high-resolution, near-infrared spectrographs (see Table 1 in Crossfield 2014) will measure abundances and planetary Huxes at multiple longitudes for short-period planets (de Kok et al. 2014). Together with low-resolution spectroscopic phase curves from HST and JWST (Stevenson et al. 2014b), this means we are entering the era of precision exocartography: such observations will constrain temperature/pressure profiles and chemistry as a function of planetary longitude. From these observations, we can soon expect global temperature and chemical abundance maps for many exoplanets' atmospheres, which will allow comparison to models of atmospheric circulation and chemistry at an unprecedented level of detail.

\subsection{The Longer Term: Beyond 2025}

A decade from now, JWST's extended mission will be near its end; Spitzer, HST, Kepler, and TESS will be long defunct; today's next-generation high-contrast imagers will have surveyed most of their accessible systems. At this point the two most-anticipated developments will be (1) the completion of one or more ground based, giant segmented-mirror telescopes (GSMTs) operating in the optical and infrared, and (2) NASA's planned WFIRSTAFTA mission, whose current design includes a highcontrast imaging system operating at optical wavelengths (Spergel et al. 2015).

The GSMTs will allow for instruments with significantly greater spectral resolution and angular resolution. With high-dispersion spectrographs, GSMTs will measure atmospheric abundances and structure and determine the signatures of atmospheric dynamics for many more planets. Such instruments will also be capable of creating global weather maps and movies of many brown dwarfs and some giant, young planets on wide orbits via Doppler Imaging (Crossfield 2014; Snellen et al. 2014). These large telescopes might even successfully detect $\mathrm{O}_{2}$ or other biosignature gases using high-dispersion spectroscopy on extrasolar planets orbiting small M dwarfs (Snellen et al. 2013, Rodler \& López-Morales 2014). Any detection would require a dedicated campaign lasting many years (Rodler \& López-Morales 2014), but such 
a result would capture the world's attention like never before.

The angular resolution provided by a GSMT's large aperture greatly expands the feasible direct-imaging science cases. That these will include young, self-luminous planets has been long appreciated (e.g., Macintosh et al. 2006). Properly designed, these ground-based imagers could access a small number of temperate super-Earths, along with substantial numbers of larger planets already known from radial velocity surveys (Crossfield 2013 Quanz et al. 2015). If high-dispersion spectroscopy is coupled to high-contrast instruments, then the dual advantages of both high-contrast and high spectral resolution would allow ELTs to probe yet fainter objects - perhaps even habitable, Earth-sized planets orbiting nearby, bright M dwarfs (Kawahara et al. 2012, Snellen et al. 2015).

A comparable but more sensitive instrument is the 2.4 m-diameter WFIRST-AFTA (Spergel et al. 2015). Though still in its early stages, current plans call for a visible-wavelength, low-resolution integral field spectrograph capable of achieving planet/star contrast levels as low as $10^{-10}$ (see Fig. 2). This facility would enable a wide range of atmospheric science including reflectedlight spectroscopy of many known planets (down to smaller sizes than could be accessed by a GSMT), and potentially even the discovery or characterization of terrestrial planets in or near the habitable zone around the nearest, most favorable FGK stars (Marley et al. 2014; Burrows 2014a; Traub et al. 2014; Greco \& Burrows 2015) - complementing the GSMT's high-contrast imaging around $\mathrm{M}$ dwarfs.

It is hoped that these ground- and space-based efforts will in turn set the stage for a large-aperture, highcontrast, optical/infrared mission in the coming decades (Kouveliotou et al. 2014, Tumlinson et al. 2015). Aside from providing the best opportunity yet to survey worlds like our own, such a program would facilitate an incredible diversity of exoplanetary science and so continue to fuel the exoplanet revolution for many years to come.

\section{Acknowledgements:}

I am grateful to J. Lothringer, Prof. T. Barman, and Dr. E. Mills for discussions and comments that substantially improved the quality of this manuscript, and to Prof. L. Close and Dr. J. Males for useful information about modern AO system performance. I also thank my referee, Prof. J. Fortney, for hist extremely useful comments and suggestions. This work was performed under contract with the California Institute of Technology/Jet Propulsion Lab funded by NASA through the Sagan Fellowship Program executed by the NASA Exoplanet Science Institute.

\section{REFERENCES}

Ackerman, A. S., \& Marley, M. S. 2001, ApJ, 556, 872

Adams, E. R., Seager, S., \& Elkins-Tanton, L. 2008, ApJ, 673, 1160

Agol, E., Cowan, N. B., Knutson, H. A., et al. 2010, ApJ, 721, 1861

Agúndez, M., Venot, O., Iro, N., et al. 2012, A\&A, 548, A73

Allard, F., Guillot, T., Ludwig, H.-G., et al. 2003, in IAU Symposium, Vol. 211, Brown Dwarfs, ed. E. Martín, 325

Apai, D., Radigan, J., Buenzli, E., et al. 2013, ApJ, 768, 121

Artigau, É., Bouchard, S., Doyon, R., \& Lafrenière, D. 2009, ApJ, 701,1534

Asplund, M., Grevesse, N., Sauval, A. J., \& Scott, P. 2009, ARA\&A, 47, 481

Atreya, S. K., \& Wong, A.-S. 2005, Space Sci. Rev., 116, 121

Bailey, J. 2014, PASA, 31, 43

Barman, T. S., Hauschildt, P. H., Schweitzer, A., et al. 2002, ApJ, 569, L51

Barman, T. S., Konopacky, Q. M., Macintosh, B., \& Marois, C. 2015, ApJ, 804, 61

Barman, T. S., Macintosh, B., Konopacky, Q. M., \& Marois, C. 2011a, ApJ, 733, 65

-. 2011b, ApJ, 735, L39

Barstow, J. K., Aigrain, S., Irwin, P. G. J., Fletcher, L. N., \& Lee, J.-M. 2013, MNRAS, 434, 2616

Barstow, J. K., Aigrain, S., Irwin, P. G. J., et al. 2014, ApJ, 786, 154

Barstow, J. K., Aigrain, S., Irwin, P. G. J., Kendrew, S., \& Fletcher, L. N. 2015, MNRAS, 448, 2546

Batalha, N., Kalirai, J., Lunine, J., Clampin, M., \& Lindler, D. 2013, Transiting Exoplanet Simulations with the James Webb Space Telescope, Tech. rep.

Bean, J. L., Miller-Ricci Kempton, E., \& Homeier, D. 2010, Nature, 468, 669

Bean, J. L., Désert, J.-M., Kabath, P., et al. 2011, ApJ, 743, 92

Beaulieu, J.-P., Tinetti, G., Kipping, D. M., et al. 2011, ApJ, 731, 16

Bechter, E. B., Crepp, J. R., Ngo, H., et al. 2014, ApJ, 788, 2

Beichman, C., Gelino, C. R., Kirkpatrick, J. D., et al. 2014a, ApJ, 783, 68

-. 2014b, ApJ, 783, 68
Beichman, C., Benneke, B., Knutson, H., et al. 2014c, PASP, 126, 1134

Beichman, C. A., Krist, J., Trauger, J. T., et al. 2010, PASP, 122, 162

Benneke, B. 2015, ArXiv e-prints, arXiv:1504.07655

Benneke, B., \& Seager, S. 2012, ApJ, 753, 100

Berta, Z. K., Charbonneau, D., Désert, J.-M., et al. 2012, ApJ, 747,35

Bétrémieux, Y., \& Kaltenegger, L. 2014, ApJ, 791, 7

Biddle, L. I., Pearson, K. A., Crossfield, I. J. M., et al. 2014, MNRAS, 443, 1810

Biller, B. A., Liu, M. C., Wahhaj, Z., et al. 2013, ApJ, 777, 160

Birkby, J. L., de Kok, R. J., Brogi, M., et al. 2013, MNRAS, 436, L35

Bonfils, X., Delfosse, X., Udry, S., et al. 2013, A\&A, 549, A109

Bonnefoy, M., Currie, T., Marleau, G.-D., et al. 2014a, A\&A, 562, A 111

Bonnefoy, M., Marleau, G.-D., Galicher, R., et al. 2014b, A\&A, 567, L9

Borysow, A., Jorgensen, U. G., \& Zheng, C. 1997, A\&A, 324, 185

Bouvier, J., Matt, S. P., Mohanty, S., et al. 2014, Protostars and Planets VI, 433

Brogi, M., de Kok, R. J., Birkby, J. L., Schwarz, H., \& Snellen, I. A. G. 2014, A\&A, 565, A124

Brogi, M., Snellen, I. A. G., de Kok, R. J., et al. 2012, Nature, 486,502

—. 2013, ApJ, 767, 27

Brown, T. M., Charbonneau, D., Gilliland, R. L., Noyes, R. W., \& Burrows, A. 2001, ApJ, 552, 699

Buenzli, E., Apai, D., Radigan, J., Reid, I. N., \& Flateau, D. 2014, ApJ, 782, 77

Burgasser, A. J. 2001, PhD thesis, Department of Physics, California Institute of Technology

Burgasser, A. J., Marley, M. S., Ackerman, A. S., et al. 2002, ApJ, 571, L151

Burrows, A. 2014a, ArXiv e-prints, arXiv:1412.6097

Burrows, A., Budaj, J., \& Hubeny, I. 2008, ApJ, 678, 1436

Burrows, A., Rauscher, E., Spiegel, D. S., \& Menou, K. 2010, ApJ, 719, 341

Burrows, A., \& Sharp, C. M. 1999, ApJ, 512, 843 
Burrows, A., Sudarsky, D., \& Lunine, J. I. 2003, ApJ, 596, 587 Burrows, A. S. 2014b, Proceedings of the National Academy of Science, 111, 12601

Chapman, S. 1930, The London, Edinburgh, and Dublin Philosophical Magazine and Journal of Science, 10, 369

Charbonneau, D., Brown, T. M., Noyes, R. W., \& Gilliland, R. L. 2002, ApJ, 568, 377

Charbonneau, D., Allen, L. E., Megeath, S. T., et al. 2005, ApJ, 626,523

Chauvin, G., Lagrange, A.-M., Dumas, C., et al. 2004, A\&A, 425, L29

Christiansen, J. L., Ballard, S., Charbonneau, D., et al. 2010, ApJ, 710, 97

Close, L. M., Males, J. R., Follette, K. B., et al. 2014, in Society of Photo-Optical Instrumentation Engineers (SPIE) Conference Series, Vol. 9148, Society of Photo-Optical Instrumentation Engineers (SPIE) Conference Series, 1

Correia, A. C. M., \& Laskar, J. 2010, Tidal Evolution of Exoplanets, ed. S. Seager, 239-266

Cowan, N. B., \& Agol, E. 2011, ApJ, 726, 82

Cowan, N. B., Agol, E., \& Charbonneau, D. 2007, MNRAS, 379, 641

Cowan, N. B., Machalek, P., Croll, B., et al. 2012, ApJ, 747, 82

Cowan, N. B., Greene, T., Angerhausen, D., et al. 2015, PASP, 127,311

Croll, B., Lafreniere, D., Albert, L., et al. 2011, AJ, 141, 30

Crossfield, I. J. M. 2013, A\&A, 551, A99

- 2014, A\&A, 566, A130

Crossfield, I. J. M., Barman, T., \& Hansen, B. M. S. 2011, ApJ, 736, 132

Crossfield, I. J. M., Barman, T., Hansen, B. M. S., Tanaka, I., \& Kodama, T. 2012a, ApJ, 760, 140

Crossfield, I. J. M., Hansen, B. M. S., \& Barman, T. 2012b, ApJ, 746,46

Crossfield, I. J. M., Hansen, B. M. S., Harrington, J., et al. 2010, ApJ, 723, 1436

Crossfield, I. J. M., Knutson, H., Fortney, J., et al. 2012c, ApJ, 752,81

Crossfield, I. J. M., Biller, B., Schlieder, J. E., et al. 2014, Nature, 505,654

Crossfield, I. J. M., Petigura, E., Schlieder, J. E., et al. 2015, ApJ, 804, 10

Crouzet, N., McCullough, P. R., Deming, D., \& Madhusudhan, N. 2014, ApJ, 795, 166

Currie, T., Burrows, A., Itoh, Y., et al. 2011, ApJ, 729, 128

Cushing, M. C., Kirkpatrick, J. D., Gelino, C. R., et al. 2011, ApJ, 743, 50

de Kok, R. J., Birkby, J., Brogi, M., et al. 2014, A\&A, 561, A150

de Kok, R. J., Brogi, M., Snellen, I. A. G., et al. 2013, A\&A, 554, A82

de Mooij, E. J. W., Brogi, M., de Kok, R. J., et al. 2012, A\&A, 538, A46

Deming, D., Seager, S., Richardson, L. J., \& Harrington, J. 2005, Nature, 434, 740

Deming, D., Seager, S., Winn, J., et al. 2009, PASP, 121, 952

Deming, D., Wilkins, A., McCullough, P., et al. 2013, ApJ, 774, 95

Demory, B.-O., Gillon, M., Madhusudhan, N., \& Queloz, D. 2015, ArXiv e-prints, arXiv:1505.00269

Demory, B.-O., de Wit, J., Lewis, N., et al. 2013, ApJ, 776, L25

Désert, J.-M., Vidal-Madjar, A., Lecavelier Des Etangs, A., et al. 2008, A\&A, 492, 585

Désert, J.-M., Bean, J., Miller-Ricci Kempton, E., et al. 2011, ApJ, 731, L40+

Diamond-Lowe, H., Stevenson, K. B., Bean, J. L., Line, M. R., \& Fortney, J. J. 2014, ApJ, 796, 66

Dong, S., \& Zhu, Z. 2013, ApJ, 778, 53

Dressing, C. D., \& Charbonneau, D. 2015, ArXiv e-prints, arXiv:1501.01623

Drossart, P., Fouchet, T., Crovisier, J., et al. 1999, in ESA Special Publication, Vol. 427, The Universe as Seen by ISO, ed. P. Cox \& M. Kessler, 169

Dupuy, T. J., \& Kraus, A. L. 2013, Science, 341, 1492

Ehrenreich, D., Bourrier, V., Bonfils, X., et al. 2012, A\&A, 547, A18

Ehrenreich, D., Bonfils, X., Lovis, C., et al. 2014, A\&A, 570, A89
Ehrenreich, D., Bourrier, V., Wheatley, P. J., et al. 2015, Nature, 522,459

Esteves, L. J., De Mooij, E. J. W., \& Jayawardhana, R. 2015, ApJ, 804, 150

Evans, T. M., Aigrain, S., Gibson, N., et al. 2015, ArXiv e-prints, arXiv:1504.05942

Evans, T. M., Pont, F., Sing, D. K., et al. 2013, ApJ, 772, L16

Fortney, J. J. 2005, MNRAS, 364, 649

Fortney, J. J., Lodders, K., Marley, M. S., \& Freedman, R. S. 2008, ApJ, 678, 1419

Fortney, J. J., Marley, M. S., \& Barnes, J. W. 2007, ApJ, 659 1661

Fortney, J. J., Mordasini, C., Nettelmann, N., et al. 2013, ApJ, 775,80

Fortney, J. J., Shabram, M., Showman, A. P., et al. 2010, ApJ, 709,1396

Fossati, L., Haswell, C. A., Linsky, J. L., \& Kislyakova, K. G. 2015, in Astrophysics and Space Science Library, Vol. 411, Astrophysics and Space Science Library, ed. H. Lammer \& M. Khodachenko, 59

Fossati, L., Haswell, C. A., Froning, C. S., et al. 2010, ApJ, 714, L222

Fraine, J., Deming, D., Benneke, B., et al. 2014, Nature, 513, 526 Fressin, F., Torres, G., Charbonneau, D., et al. 2013, ApJ, 766, 81

Gagné, J., Lafrenière, D., Doyon, R., Malo, L., \& Artigau, É. 2014, ApJ, 783, 121

Gibson, N. P., Aigrain, S., Barstow, J. K., et al. 2013, MNRAS, 428,3680

Gibson, N. P., Pont, F., \& Aigrain, S. 2011, MNRAS, 411, 2199

Glauser, A. M., van Boekel, R., Krause, O., et al. 2013, Journal of Astronomical Instrumentation, 2, 50004

Goukenleuque, C., Bézard, B., Joguet, B., Lellouch, E., \&

Freedman, R. 2000, Icarus, 143, 308

Greco, J. P., \& Burrows, A. 2015, ArXiv e-prints, arXiv:1505.07832

Grillmair, C. J., Burrows, A., Charbonneau, D., et al. 2008 , Nature, 456, 767

Hansen, C. J., Schwartz, J. C., \& Cowan, N. B. 2014, MNRAS, 444,3632

Hardy, J. W. 1998, Adaptive Optics for Astronomical Telescopes

Haswell, C. A., Fossati, L., Ayres, T., et al. 2012, ApJ, 760, 79

Haynes, K., Mandell, A. M., Madhusudhan, N., Deming, D., \& Knutson, H. 2015, ApJ, 806, 146

Helling, C., Dehn, M., Woitke, P., \& Hauschildt, P. H. 2008, ApJ, 675, L105

Heng, K. 2012, ApJ, 761, L1

Heng, K., Lyons, J. R., \& Tsai, S.-M. 2015, ArXiv e-prints, arXiv:1506.05501

Heng, K., \& Showman, A. P. 2015, Annual Review of Earth and Planetary Sciences, 43, 509

Hoeijmakers, H. J., de Kok, R. J., Snellen, I. A. G., et al. 2015, A\&A, 575, A20

Howard, A. W., Marcy, G. W., Bryson, S. T., et al. 2012, ApJS, 201, 15

Howe, A. R., \& Burrows, A. S. 2012, ApJ, 756, 176

Howell, S. B., Sobeck, C., Haas, M., et al. 2014, PASP, 126, 398

Hu, R. 2014, ArXiv e-prints, arXiv:1412.7582

Hu, R., Seager, S., \& Yung, Y. L. 2015, ArXiv e-prints, arXiv:1505.02221

Hubbard, W. B., Fortney, J. J., Lunine, J. I., et al. 2001, ApJ, 560,413

Hubeny, I., Burrows, A., \& Sudarsky, D. 2003, ApJ, 594, 1011

Irwin, P. G. J., Teanby, N. A., de Kok, R., et al. 2008, J. Quant. Spec. Radiat. Transf., 109, 1136

Janson, M., Brandt, T. D., Kuzuhara, M., et al. 2013, ApJ, 778, L4

Kaltenegger, L., \& Traub, W. A. 2009, ApJ, 698, 519

Karkoschka, E., \& Tomasko, M. G. 2011, Icarus, 211, 780

Kawahara, H., Matsuo, T., Takami, M., et al. 2012, ApJ, 758, 13

Kempton, E. M.-R., Perna, R., \& Heng, K. 2014, ApJ, 795, 24

Kim, S. J., Geballe, T. R., \& Noll, K. S. 2000, Icarus, 147, 588

Kirkpatrick, J. D., Reid, I. N., Liebert, J., et al. 1999, ApJ, 519, 802

Kirkpatrick, J. D., Gelino, C. R., Cushing, M. C., et al. 2012, ApJ, 753, 156

Knapp, G. R., Leggett, S. K., Fan, X., et al. 2004, AJ, 127, 3553 
Knutson, H. A., Benneke, B., Deming, D., \& Homeier, D. 2014a, Nature, 505, 66

Knutson, H. A., Charbonneau, D., Allen, L. E., Burrows, A., \& Megeath, S. T. 2008, ApJ, 673, 526

Knutson, H. A., Howard, A. W., \& Isaacson, H. 2010, ApJ, 720, 1569

Knutson, H. A., Charbonneau, D., Allen, L. E., et al. 2007, Nature, 447, 183

Knutson, H. A., Madhusudhan, N., Cowan, N. B., et al. 2011, ApJ, 735, 27

Knutson, H. A., Lewis, N., Fortney, J. J., et al. 2012, ApJ, 754, 22

Knutson, H. A., Dragomir, D., Kreidberg, L., et al. 2014b, ApJ, 794,155

Konopacky, Q. M., Barman, T. S., Macintosh, B. A., \& Marois, C. 2013, Science, 339, 1398

Kopytova, T. G., Crossfield, I. J. M., Deacon, N. R., et al. 2014, ApJ, 797, 3

Koskinen, T. T., Harris, M. J., Yelle, R. V., \& Lavvas, P. 2013, Icarus, 226, 1678

Kostov, V., \& Apai, D. 2013, ApJ, 762, 47

Kouveliotou, C., Agol, E., Batalha, N., et al. 2014, ArXiv e-prints, arXiv:1401.3741

Kreidberg, L., Bean, J. L., Désert, J.-M., et al. 2014a, ApJ, 793, L27

-. 2014b, Nature, 505, 69

Kreidberg, L., Line, M. R., Bean, J. L., et al. 2015, ArXiv e-prints, arXiv:1504.05586

Kuzuhara, M., Tamura, M., Kudo, T., et al. 2013, ApJ, 774, 11

Lammer, H., Erkaev, N. V., Odert, P., et al. 2013, MNRAS, 430, 1247

Lanotte, A. A., Gillon, M., Demory, B.-O., et al. 2014, A\&A, 572, A73

Lavvas, P., Koskinen, T., \& Yelle, R. V. 2014, ApJ, 796, 15

Lecavelier Des Etangs, A., Pont, F., Vidal-Madjar, A., \& Sing, D. 2008a, A\&A, 481, L83

Lecavelier Des Etangs, A., Vidal-Madjar, A., Désert, J., \& Sing, D. 2008b, A\&A, 485, 865

Lee, J.-M., Heng, K., \& Irwin, P. G. J. 2013, ApJ, 778, 97

Lee, J.-M., Irwin, P. G. J., Fletcher, L. N., Heng, K., \& Barstow, J. K. 2014, ApJ, 789, 14

Leggett, S. K., Morley, C. V., Marley, M. S., et al. 2013, ApJ, 763,130

Line, M. R., Fortney, J. J., Marley, M. S., \& Sorahana, S. 2014a, ApJ, 793, 33

Line, M. R., Knutson, H., Wolf, A. S., \& Yung, Y. L. 2014b, ApJ, 783,70

Line, M. R., Teske, J., Burningham, B., Fortney, J., \& Marley, M. 2015, ArXiv e-prints, arXiv:1504.06670

Line, M. R., Vasisht, G., Chen, P., Angerhausen, D., \& Yung, Y. L. 2011, ApJ, 738, 32

Line, M. R., \& Yung, Y. L. 2013, ApJ, 779, 3

Line, M. R., Wolf, A. S., Zhang, X., et al. 2013, ApJ, 775, 137

Linsky, J. L., Yang, H., France, K., et al. 2010, ApJ, 717, 1291

Lockwood, A. C., Johnson, J. A., Bender, C. F., et al. 2014, ApJ, 783, L29

Lodders, K. 1999, ApJ, 519, 793

Lodders, K., \& Fegley, Jr., B. 2006, Chemistry of Low Mass Substellar Objects, ed. J. W. Mason, 1

Luhman, K. L. 2014, The Astrophysical Journal Letters, 786, L18

Lunine, J. I., Hubbard, W. B., \& Marley, M. S. 1986, ApJ, 310, 238

Macintosh, B., Troy, M., Doyon, R., et al. 2006, in Society of Photo-Optical Instrumentation Engineers (SPIE) Conference Series, Vol. 6272, Society of Photo-Optical Instrumentation Engineers (SPIE) Conference Series

Macintosh, B., Graham, J. R., Ingraham, P., et al. 2014, Proceedings of the National Academy of Science, 111, 12661

Madhusudhan, N. 2012, ApJ, 758, 36

Madhusudhan, N., Burrows, A., \& Currie, T. 2011a, ApJ, 737, 34

Madhusudhan, N., Knutson, H., Fortney, J. J., \& Barman, T. 2014, Protostars and Planets VI, 739

Madhusudhan, N., \& Seager, S. 2009, ApJ, 707, 24

-. 2010, ApJ, 725, 261

-. 2011, ApJ, 729, 41

Madhusudhan, N., Harrington, J., Stevenson, K. B., et al. 2011b, Nature, 469, 64
Mandell, A. M., Drake Deming, L., Blake, G. A., et al. 2011, ApJ, 728,18

Marcy, G., Butler, R. P., Fischer, D., et al. 2005, Progress of Theoretical Physics Supplement, 158, 24

Marley, M., Lupu, R., Lewis, N., et al. 2014, ArXiv e-prints, arXiv: 1412.8440

Marley, M. S., Ackerman, A. S., Cuzzi, J. N., \& Kitzmann, D. 2013, Clouds and Hazes in Exoplanet Atmospheres, ed. S. J. Mackwell, A. A. Simon-Miller, J. W. Harder, \& M. A. Bullock, 367-391

Marley, M. S., Fortney, J., Seager, S., \& Barman, T. 2007, Protostars and Planets V, 733

Marley, M. S., \& Robinson, T. D. 2014, ArXiv e-prints, arXiv:1410.6512

Marley, M. S., Saumon, D., \& Goldblatt, C. 2010, ApJ, 723, L117

Marois, C., Lafrenière, D., Doyon, R., Macintosh, B., \& Nadeau, D. 2006, ApJ, 641, 556

Marois, C., Macintosh, B., Barman, T., et al. 2008, Science, 322, 1348

Martín, E. L., Delfosse, X., Basri, G., et al. 1999, AJ, 118, 2466

Mawet, D., Pueyo, L., Lawson, P., et al. 2012, in Society of Photo-Optical Instrumentation Engineers (SPIE) Conference Series, Vol. 8442, Society of Photo-Optical Instrumentation Engineers (SPIE) Conference Series

McCullough, P. R., Crouzet, N., Deming, D., \& Madhusudhan, N. 2014, ApJ, 791, 55

Metchev, S. A., Heinze, A., Apai, D., et al. 2015, ApJ, 799, 154

Miguel, Y., \& Kaltenegger, L. 2014, ApJ, 780, 166

Miller-Ricci, E., Seager, S., \& Sasselov, D. 2009, ApJ, 690, 1056

Miller-Ricci Kempton, E., \& Rauscher, E. 2012, ApJ, 751, 117

Miller-Ricci Kempton, E., Zahnle, K., \& Fortney, J. J. 2012, ApJ, 745,3

Montet, B. T., Morton, T. D., Foreman-Mackey, D., et al. 2015, ArXiv e-prints, arXiv:1503.07866

Mordasini, C., Alibert, Y., Georgy, C., et al. 2012, A\&A, 547, A112

Morello, G., Waldmann, I. P., Tinetti, G., et al. 2015, ApJ, 802, 117

Morley, C. V., Fortney, J. J., Kempton, E. M.-R., et al. 2013, ApJ, 775, 33

Morley, C. V., Fortney, J. J., Marley, M. S., et al. 2012, ApJ, 756,172

Morley, C. V., Marley, M. S., Fortney, J. J., et al. 2014, ApJ, 787,78

Moses, J. I., Visscher, C., Fortney, J. J., et al. 2011, ApJ, 737, 15

Moses, J. I., Line, M. R., Visscher, C., et al. 2013, ApJ, 777, 34

Mulders, G. D., Pascucci, I., \& Apai, D. 2015, ApJ, 798, 112

Murray-Clay, R. A., Chiang, E. I., \& Murray, N. 2009, ApJ, 693, 23

Nascimbeni, V., Piotto, G., Pagano, I., et al. 2013, A\&A, 559, A32

Nikolov, N., Sing, D. K., Burrows, A. S., et al. 2015, MNRAS, 447,463

Öberg, K. I., Murray-Clay, R., \& Bergin, E. A. 2011, ApJ, 743, L16

Orton, G. S. 1981, in IAU Symposium, Vol. 96, Infrared Astronomy, ed. C. G. Wynn-Williams \& D. P. Cruikshank, $35-55$

Oshagh, M., Santos, N. C., Ehrenreich, D., et al. 2014, A\&A, 568, A99

Owen, J. E., \& Jackson, A. P. 2012, MNRAS, 425, 2931

Parmentier, V., Showman, A. P., \& Lian, Y. 2013, A\&A, 558, A91

Patience, J., King, R. R., de Rosa, R. J., \& Marois, C. 2010, A\&A, 517, A76

Penz, T., Erkaev, N. V., Kulikov, Y. N., et al. 2008, P. \& S.S., 56, 1260

Perez-Becker, D., \& Showman, A. P. 2013, ApJ, 776, 134

Perna, R., Menou, K., \& Rauscher, E. 2010, ApJ, 719, 1421

Perryman, M., Hartman, J., Bakos, G. Á., \& Lindegren, L. 2014, ApJ, 797, 14

Pont, F., Sing, D. K., Gibson, N. P., et al. 2013, MNRAS, 432, 2917

Prinn, R. G., \& Barshay, S. S. 1977, Science, 198, 1031

Quanz, S. P., Crossfield, I., Meyer, M. R., Schmalzl, E., \& Held, J. 2015, International Journal of Astrobiology, 14, 279

Radigan, J., Lafrenière, D., Jayawardhana, R., \& Artigau, E. 2014, ApJ, 793, 75 
Rauscher, E., \& Kempton, E. M. R. 2014, ApJ, 790, 79

Rauscher, E., \& Menou, K. 2013, ApJ, 764, 103

Rauscher, E., Menou, K., Cho, J. Y.-K., Seager, S., \& Hansen, B. M. S. 2007, ApJ, 662, L115

Redfield, S., Endl, M., Cochran, W. D., \& Koesterke, L. 2008, ApJ, 673, L87

Reid, I. N., \& Hawley, S. L. 2005, New light on dark stars : red dwarfs, low-mass stars, brown dwarfs, doi:10.1007/3-540-27610-6

Ricker, G. R., Winn, J. N., Vanderspek, R., et al. 2014, in Society of Photo-Optical Instrumentation Engineers (SPIE) Conference Series, Vol. 9143, Society of Photo-Optical Instrumentation Engineers (SPIE) Conference Series, 20

Robinson, T. D., \& Catling, D. C. 2014, Nature Geoscience, 7, 12

Robinson, T. D., \& Marley, M. S. 2014, ApJ, 785, 158

Rodler, F., Kürster, M., \& Barnes, J. R. 2013, MNRAS, 432, 1980

Rodler, F., \& López-Morales, M. 2014, ApJ, 781, 54

Rodler, F., Lopez-Morales, M., \& Ribas, I. 2012, ApJ, 753, L25

Rosenthal, E. D., Gurwell, M. A., \& Ho, P. T. P. 1996, Nature, 384,243

Rowe, J. F., Matthews, J. M., Seager, S., et al. 2008, ApJ, 689, 1345

Sayanagi, K. M., Dyudina, U. A., Ewald, S. P., et al. 2013, Icarus, 223,460

Schwartz, J. C., \& Cowan, N. B. 2015, MNRAS, 449, 4192

Seager, S., \& Deming, D. 2010, ARA\&A, 48, 631

Seager, S., \& Sasselov, D. D. 1998, ApJ, 502, L157

-. 2000, ApJ, 537, 916

Sharp, C. M., \& Burrows, A. 2007, ApJS, 168, 140

Showman, A. P., Fortney, J. J., Lian, Y., et al. 2009, ApJ, 699, 564

Showman, A. P., \& Guillot, T. 2002, A\&A, 385, 166

Showman, A. P., \& Kaspi, Y. 2013, ApJ, 776, 85

Shporer, A., \& Hu, R. 2015, ArXiv e-prints, arXiv:1504.00498

Sing, D. K., Désert, J., Lecavelier Des Etangs, A., et al. 2009 , A\&A, 505, 891

Sing, D. K., Lecavelier des Etangs, A., Fortney, J. J., et al. 2013, MNRAS, 436, 2956

Sing, D. K., Wakeford, H. R., Showman, A. P., et al. 2015, MNRAS, 446, 2428

Skemer, A. J., Marley, M. S., Hinz, P. M., et al. 2014a, ApJ, 792, 17

Skemer, A. J., Hinz, P., Esposito, S., et al. 2014b, in Society of Photo-Optical Instrumentation Engineers (SPIE) Conference Series, Vol. 9148, Society of Photo-Optical Instrumentation Engineers (SPIE) Conference Series, 0

Smith, W. H. 1987, PASP, 99, 1344

Snellen, I., de Kok, R., Birkby, J. L., et al. 2015, A\&A, 576, A59

Snellen, I. A. G., Brandl, B. R., de Kok, R. J., et al. 2014, Nature, 509, 63

Snellen, I. A. G., de Kok, R. J., de Mooij, E. J. W., \& Albrecht, S. 2010, Nature, 465, 1049

Snellen, I. A. G., de Kok, R. J., le Poole, R., Brogi, M., \& Birkby, J. 2013, ApJ, 764, 182

Sozzetti, A., Giacobbe, P., Lattanzi, M. G., et al. 2014, MNRAS, 437,497

Sparks, W. B., \& Ford, H. C. 2002, ApJ, 578, 543

Spergel, D., Gehrels, N., Baltay, C., et al. 2015, ArXiv e-prints, arXiv: 1503.03757

Sromovsky, L. A., Baines, K. H., \& Fry, P. M. 2013, Icarus, 226, 402
Stephens, D. C., Leggett, S. K., Cushing, M. C., et al. 2009, ApJ, 702,154

Stevenson, K. B., Bean, J. L., Madhusudhan, N., \& Harrington, J. 2014a, ApJ, 791, 36

Stevenson, K. B., Harrington, J., Nymeyer, S., et al. 2010, Nature, 464, 1161

Stevenson, K. B., Harrington, J., Lust, N. B., et al. 2012, ApJ, 755,9

Stevenson, K. B., Désert, J.-M., Line, M. R., et al. 2014b, Science, 346, 838

Sudarsky, D., Burrows, A., \& Pinto, P. 2000, ApJ, 538, 885

Swain, M., Deroo, P., Tinetti, G., et al. 2013, Icarus, 225, 432

Swain, M. R. 2012, in American Astronomical Society Meeting Abstracts, Vol. 220, American Astronomical Society Meeting Abstracts \#220, 505.05

Swain, M. R., Vasisht, G., Henning, T., Tinetti, G., \& Beaulieu, J.-P. 2010a, in Society of Photo-Optical Instrumentation Engineers (SPIE) Conference Series, Vol. 7731, Society of Photo-Optical Instrumentation Engineers (SPIE) Conference Series, 25

Swain, M. R., Vasisht, G., \& Tinetti, G. 2008, Nature, 452, 329

Swain, M. R., Deroo, P., Griffith, C. A., et al. 2010b, Nature, 463, 637

Testi, L. 2009, A\&A, 503, 639

Tinetti, G., Drossart, P., Eccleston, P., et al. 2015, ArXiv e-prints, arXiv:1502.05747

Todorov, K. O., Deming, D., Burrows, A., \& Grillmair, C. J. 2014, ApJ, 796, 100

Todorov, K. O., Line, M. R., Pineda, J. E., et al. 2015, ArXiv e-prints, arXiv:1504.00217

Traub, W. A., Belikov, R., Guyon, O., et al. 2014, in Society of Photo-Optical Instrumentation Engineers (SPIE) Conference Series, Vol. 9143, Society of Photo-Optical Instrumentation Engineers (SPIE) Conference Series, 0

Tsuji, T., \& Nakajima, T. 2003, ApJ, 585, L151

Tsuji, T., Nakajima, T., \& Yanagisawa, K. 2004, ApJ, 607, 511

Tumlinson, J., Seager, S., Dalcanton, J., et al. 2015, in American Astronomical Society Meeting Abstracts, Vol. 225, American Astronomical Society Meeting Abstracts, 338.19

Valencia, D., Ikoma, M., Guillot, T., \& Nettelmann, N. 2010, A\&A, 516, A20

Vanderburg, A., Montet, B. T., Johnson, J. A., et al. 2015, ApJ, 800,59

Vidal-Madjar, A., Lecavelier des Etangs, A., Désert, J.-M., et al. 2003, Nature, 422, 143

Vidal-Madjar, A., Désert, J.-M., Lecavelier des Etangs, A., et al. 2004, ApJ, 604, L69

Visscher, C., Lodders, K., \& Fegley, Jr., B. 2006, ApJ, 648, 1181

Wakeford, H. R., \& Sing, D. K. 2015, A\&A, 573, A122

Waldmann, I. P., Tinetti, G., Drossart, P., et al. 2012, ApJ, 744, 35

Walker, G. A. H., Croll, B., Matthews, J. M., et al. 2008, A\&A, 482,691

Webber, M. W., Lewis, N. K., Marley, M., et al. 2015, ArXiv e-prints, arXiv:1503.01028

Wong, I., Knutson, H. A., Lewis, N. K., et al. 2015, ArXiv e-prints, arXiv:1505.03158

Wyttenbach, A., Ehrenreich, D., Lovis, C., Udry, S., \& Pepe, F. 2015, A\&A, 577, A62

Zellem, R. T., Griffith, C. A., Deroo, P., Swain, M. R., \& Waldmann, I. P. 2014a, ApJ, 796, 48

Zellem, R. T., Lewis, N. K., Knutson, H. A., et al. 2014b, ApJ, 790, 53 